\title{
Flow-Heat-Solid Coupling Thermal Deformation Analysis of Hydrostatic Spindle under Viscosity Temperature Effect
}

Dongju Chen ( $\sim$ djchen@bjut.edu.cn )

Beijing University of Technology https://orcid.org/0000-0003-3839-4079

Xuan Zhang

Beijing University of Technology

\section{Original Article}

Keywords: Viscosity-temperature effect, Hydrostatic bearing, Thermal deformation, Coupling analysis

Posted Date: May 18th, 2021

DOI: https://doi.org/10.21203/rs.3.rs-500698/v1

License: (c) (1) This work is licensed under a Creative Commons Attribution 4.0 International License.

Read Full License 


\title{
Flow-Heat-Solid Coupling Thermal Deformation Analysis of Hydrostatic Spindle under Viscosity Temperature Effect
}

\author{
Dongju Chen*, Xuan Zhang
}

\begin{abstract}
The hydrostatic bearing oil film plays a key role in supporting and lubricating. As the speed increases, the temperature of the lubricating oil increases and the viscosity decreases. As a result, the bearing capacity of the oil film is reduced, which affects the motion accuracy of the hydrostatic bearing. In this paper, the simulation and analysis of the temperature rise of the hydrostatic bearing oil film under the constant viscosity and the viscosity-temperature effect are performed respectively. Then, based on the fluid-heat-solid coupling analysis theory, the temperature field of the hydrostatic bearing and the thermal deformation of the spindle shaft with and without the viscosity-temperature effect are analyzed separately. The temperature field of the shaft and the thermal deformation of the spindle shaft are analyzed separately. Finally, the bearing temperature and shaft deformation are compared with the experimental values for error analysis. It is found that the error rate is smaller when the viscosity-temperature effect is considered. Considering the viscosity-temperature effect, the maximum error rates of the temperature of the radial and thrust bearing bushes are $11.05 \%$ and $7.82 \%$, and the maximum error rates of the thermal deformation of the spindle shaft in the axial and radial directions are $12.03 \%$ and $18.57 \%$.
\end{abstract}

Keywords: Viscosity-temperature effect, Hydrostatic bearing, Thermal deformation, Coupling analysis

\section{Introduction}

Hydrostatic spindle including bearings in radial and axial direction, oil film and shaft, when it works, the temperature of the oil film between bearing and shaft will increase, thermal deformation will occur[1], and this will influence the motion accuracy of the spindle. During work process, the viscosity of the oil film is changed in the work process, in tradition research this is seen as constant[2], which will have an impact on the final result. In addition, the mechanism of thermal interaction between fluid and solid is not clear yet. The neglection of thermal-structure interaction of high-speed spindle system may lead to the modeling error of the thermal characteristics[3]. Lu et al. [4] performed a one-way fluid-solid coupling analysis on a hydrostatic piezoelectric spindle, and the influence of the structure deformation on the static performance of the hydrostatic piezoelectric spindle is studied. Yu Mubing et al. [5] studied the thermo-fluid-solid coupling deformation and friction failure mechanisms of bearing friction pairs under highspeed and heavy-load conditions. The results show that the

\footnotetext{
*Correspondence: caffeezhang@hotmail.com

Mechanical Industry Key Laboratory of Heavy Machine Tool Digital Design and Testing, Faculty of Materials and Manufacturing, Beijing University of Technology, Beijing 100124, China.
}

temperature of the oil film rised sharply, the viscosity of the lubricating oil decreased rapidly, and the deformation increased. Feng Tan et al. [6] used a constraint optimization problem to obtain more accurate convective heat transfer coefficients. Zhang Yifan et al. [7] developed an active cooling strategy for spindle thermal balance control to accurately eliminate disturbing heat transfer. Wang et al. [8] used computational fluid dynamics software FLUENT to study hydrostatic and hydrostatic bearings for ultra-highspeed grinding, obtained the oil film temperature distribution under different input conditions, and found that the spindle speed is the most important factor affecting the temperature distribution. Shao Junpeng et al. [9] proposed a new wedge-shaped structure that opens at the edge of the oil seal, using dynamic pressure to make up for the loss and deficiency of the hydrostatic bearing capacity, thereby achieving hydrostatic lubrication. Xiang Sitong et al. [10] proposed the analysis of main axis thermal error. In order to avoid these contradictions, a new data-driven prediction (DDP) method is proposed for dynamic linear modeling of thermal error of the spindle. Yan Ke et al. [11] took into 
account the thermal-structural interaction and developed a network method for spindle transient analysis. T. Holkup et al. [12] established a thermal-structure model of the electric spindle, which can predict the temperature field distribution and temperature rise of the electric spindle. Su Hao et al. [13] established a hydrostatic spindle system model through FVEM to predict the change in thermal behavior of the hydrostatic spindle system.Qingyu. Meng et al. [14] analyzed various fractal parameters influenced on thermal resistance network model of motorized spindle, and $\mathrm{Wu}$ Chengyang et al. [15] used a deep learning method to predict thermal error of spindle, this learning model has good applicability to the spindle thermal error prediction and compensation. In addition to the modeling and impact study of the spindle thermal, Jialan liu et al. [16] also compensates for the thermally-induced error of the spindle based on long short term memory neural networks.

When the above scholars study the temperature rise and deformation of the hydrostatic spindle, most scholars take the viscosity value of lubricating oil as a constant value for analysis. In this paper, based on the hydrostatic spindle testbed, the viscosity temperature equation is introduced to simulate the real-time variation of lubricating oil viscosity with temperature, and then the oil film temperature rise under the viscosity temperature effect is simulated, which is compared with the oil film temperature rise without considering the viscosity temperature effect. The influence of oil film with and without viscosity temperature effect on the temperature field and thermal deformation of bearing bush and spindle shaft is analyzed. The temperature field and thermal deformation are compared and analyzed.

\section{Simulation and Experiment}

2.1 Theoretical calculation of oil film temperature rise of hydrostatic bearing

\subsubsection{Structure and working principle of hydrostatic bearing}

According to the different structure and load-bearing methods of hydrostatic bearings, the hydrostatic bearing is divided into a radial bearing and a thrust bearing. The radial bearing bears the load in the radial direction of the spindle, and the thrust bearing bears the load in the axial direction of the spindle. In the hydrostatic spindle system, the positioning of the spindle is achieved through the cooperation of radial bearings and thrust bearings. The radial bearing is a four-cavity structure, the thrust bearing is a six-cavity structure, and bearing is throttled by small holes. Figure 1 is a schematic diagram of the structure of a hydrostatic bearing, and Table 1 is a structural parameter of the hydrostatic bearing.
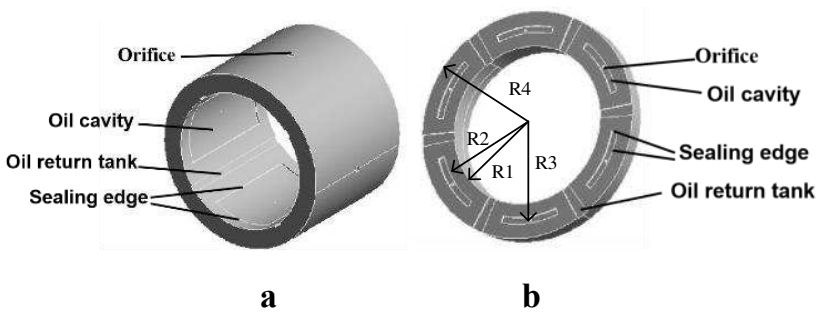

FIgure 1 Structure of hydrostatic bearing: (a) Radial bearing, (b) Thrust bearing

Table 1 Structural parameters of hydrostatic bearing

\begin{tabular}{|c|c|c|c|c|c|c|c|}
\hline \multicolumn{4}{|c|}{ Radial bearing } & \multicolumn{4}{|c|}{ Thrust bearing } \\
\hline Flow Coefficient & 0.7 & $\begin{array}{l}\text { Angle of oil cavity } \\
\left({ }^{\circ}\right)\end{array}$ & 54 & $\begin{array}{c}\text { Bearing inner } \\
\text { diameter } \mathrm{R} 1(\mathrm{~mm})\end{array}$ & 35 & $\begin{array}{c}\text { Oil chamber angle } \\
\left({ }^{\circ}\right)\end{array}$ & $18^{\circ}$ \\
\hline $\begin{array}{l}\text { Bearing length } \\
\quad(\mathrm{mm})\end{array}$ & 70 & $\begin{array}{l}\text { Oil cavity length } \\
(\mathrm{mm})\end{array}$ & 56 & $\begin{array}{l}\text { Seal oil edge } \\
\text { inner diameter } \\
\mathrm{R} 2(\mathrm{~mm})\end{array}$ & 41 & $\begin{array}{l}\text { Half angle of oil } \\
\text { cushion } \\
\left({ }^{\circ}\right)\end{array}$ & $27^{\circ}$ \\
\hline $\begin{array}{l}\text { Spindle radius } \\
(\mathrm{mm})\end{array}$ & 35 & $\begin{array}{l}\text { Oil film thickness } \\
\text { (mm) }\end{array}$ & 0.01 & $\begin{array}{l}\text { Seal oil edge } \\
\text { outer diameter } \\
\text { R3(mm) }\end{array}$ & 44 & $\begin{array}{l}\text { Lubricant density } \\
\qquad(\mathrm{kg} / \mathrm{m} 3)\end{array}$ & 858 \\
\hline $\begin{array}{l}\text { Oil seal edge } \\
\text { length factor }\end{array}$ & 0.8 & $\begin{array}{l}\text { Radial oil cavity } \\
\text { length factor }\end{array}$ & 1.6 & $\begin{array}{l}\text { Bearing outer } \\
\text { diameter } \\
\text { R4(mm) }\end{array}$ & 50.5 & $\begin{array}{l}\text { Lubricant viscosity } \\
\qquad(\text { Pa.s) }\end{array}$ & 0.00575 \\
\hline $\begin{array}{l}\text { Throttle diameter } \\
\qquad(\mathrm{mm})\end{array}$ & 0.8 & $\begin{array}{l}\text { Half angle of oil } \\
\text { cushion }\left({ }^{\circ}\right)\end{array}$ & 42 & $\begin{array}{l}\text { Oil film thickness } \\
\qquad(\mathrm{mm})\end{array}$ & 0.012 & $\begin{array}{c}\text { Specific heat capacity of } \\
\text { lubricant }\left(\mathrm{J} / \mathrm{kg} \cdot{ }^{\circ} \mathrm{C}\right)\end{array}$ & 2000 \\
\hline $\begin{array}{l}\text { Supply pressure } \\
(\mathrm{MPa})\end{array}$ & 1.8 & & & $\begin{array}{l}\text { Orifice diameter } \\
\qquad(\mathrm{mm})\end{array}$ & 1.2 & $\begin{array}{c}\text { Thermal conductivity of } \\
\text { lubricating oil } \\
\left(\mathrm{W} / \mathrm{m} \cdot{ }^{\circ} \mathrm{C}\right)\end{array}$ & 0.144 \\
\hline
\end{tabular}




\subsubsection{Radial oil film temperature rise calculation}

The frictional power $N_{t}$ of hydrostatic bearing oil film is calculated according to Newton shear stress formula:

$$
N_{t}=4 F_{t} \times \mu=4 \mu\left[\frac{A_{1}}{h_{0}}+\frac{A_{2}}{h_{0}+z_{1}}\right]\left(\frac{\pi n d}{60}\right)^{2}
$$

$\mu$ is the kinematic viscosity of lubricating oil, $A_{1}$ is the seal area of oil cavity, $A_{2}$ is the area of oil cavity, $u$ is the linear speed of shaft, $\mathrm{h}_{0}$ is the clearance of shaft radius, $\mathrm{Z}_{1}$ is the depth of radial bearing oil cavity.

Output power of oil pump

$$
N_{p}=p_{p} \cdot Q_{p}
$$

$P_{p}$ is the rated oil supply pressure of the oil pump, $P_{s}$ is the external oil supply pressure, $\lambda$ is the liquid resistance ratio of the lubricating oil, $Q_{P}$ is the flow of the radial bearing.

The radial bearing has four oil chambers, so the flow of the radial bearing is:

the radial bearing is:

$$
\begin{gathered}
Q_{P}=4 Q_{P} \\
Q_{1}=K_{0} \frac{\pi d_{0}^{2}}{4} \sqrt{\frac{2\left(P_{s}-P_{b 0}\right)}{\rho}} \\
\lambda=\frac{1}{2}\left(\sqrt{\frac{8 \rho r^{2} h_{0}^{6}\left(\frac{b}{w}+\theta\right)^{2}}{9 \pi^{2} \mu^{2} L^{2} K_{0}^{2} d_{0}^{4}} P_{s}+1}-1\right)
\end{gathered}
$$

$\mathrm{K}_{0}$ is the flow coefficient, $\mathrm{Pb} 0$ is the oil cavity pressure, $\mathrm{b}$ is the oil cavity length coefficient, $\mathrm{w}$ is the axial seal oil surface length coefficient, $\mathrm{L}$ is the axial seal oil surface length.

The temperature rise of the radial bearing oil film is composed of the frictional power of the oil film and the power consumed by the oil pump. The temperature rise of the radial oil film is calculated as:

$$
\triangle \mathrm{t}=\frac{N_{t}+N_{p}}{\rho c Q_{\mathrm{p}}}
$$

\subsubsection{Calculation of thrust oil film temperature rise:}

When calculating the temperature rise of the hydrostatic thrust bearing oil film, a simplification is performed for each oil pad of the thrust bearing model, as shown in Figure 2, the oil sealing surface is divided into five areas and calculated separately.

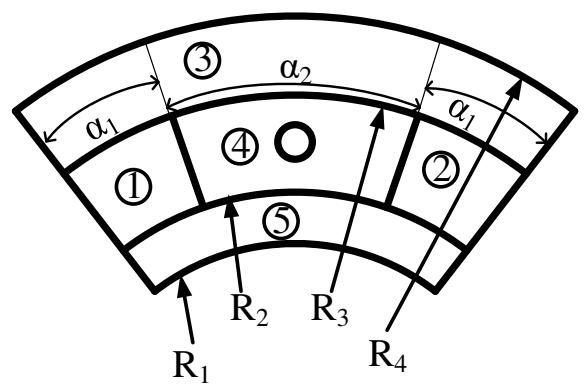

FIgure 2 Simplified model of thrust bearing seal surface

Solve the oil film friction power of five divided areas :

$$
\begin{gathered}
N_{f 1}=N_{f 2}=\int_{R 2}^{R 3} \mu\left(\frac{2 \pi R n}{60}\right)^{2}\left(\frac{\left(R_{3}-R_{2}\right) \alpha_{1} R_{3}}{h_{0}}\right) d R \\
N_{f 3}=\int_{R 3}^{R 4} \mu\left(\frac{2 \pi R n}{60}\right)^{2}\left(\frac{R\left(\alpha_{2}+2 \alpha_{1}\right)}{h_{0}}\right) d R \\
N_{f 4}=\int_{R 2}^{R 3} \mu\left(\frac{2 \pi R n}{60}\right)^{2}\left(\frac{R \alpha_{2}}{h_{0}+z}\right) d R \\
N_{f 5}=\int_{R 1}^{R 2} \mu\left(\frac{2 \pi R n}{60}\right)^{2}\left(\frac{R\left(\alpha_{2}+2 \alpha_{1}\right)}{h_{0}}\right) d R
\end{gathered}
$$

The friction power of the thrust bearing oil film is:

$$
N_{f}=6\left(N_{f 1}+N_{f 2}+N_{f 3}+N_{f 4}+N_{f 5}\right)
$$

The thrust bearing oil pump power calculation is the same as the radial oil pump power calculation method.

Oil pump output power of thrust bearing:

$$
N_{r}=p_{p} \cdot Q_{r}
$$

The temperature rise of the thrust bearing oil film is:

$$
\triangle \mathrm{t}=\frac{N_{f}+N_{r}}{\rho c Q_{\mathrm{r}}}
$$

\subsection{Simulation of oil film temperature field of hydrostatic} bearing 


\subsubsection{Visco-temperature effect and boundary conditions}

The viscosity change of lubricating oil with temperature is called the viscosity-temperature effect of lubricating oil. The hydrostatic bearing oil film plays a key role in support and lubricate. In the actual working process, as the speed increases, the oil film temperature increases, and the viscosity of the lubricating oil will change, which will affect the thermal deformation and lubrication of the hydrostatic bearing bush and the spindle shaft. Viscosity temperature equation:

$$
\mu=7.045 \mathrm{e}^{-0.02441(\mathrm{X}+T)}
$$

It can be seen from the viscosity temperature equation 15 that when the viscosity value is large, the temperature is low, while when the viscosity value is small, the temperature is high.

$\mu$ is the dynamic viscosity value at temperature $\mathrm{x}, \mathrm{X}$ is the temperature rise of lubricating oil, $\mathrm{T}$ is the Reference temperature, $\mathrm{T}=273.15 \mathrm{~K}$.

\subsection{2 boundary conditions}

When setting the oil film boundary conditions, the laminar flow model is adopted in software. The simulated oil film temperature field takes energy into account, so it is necessary to open the energy control equation in the software. The entrance boundary is the orifice of oil film, and the inlet pressure is $1.8 \mathrm{MPa}$. The outlet boundary is the sealing edge of the oil film, and the outlet pressure is $0 \mathrm{MPa}$. Considering the thermal effect, the outlet temperature is $305 \mathrm{~K}$. Other settings remain default.

When setting the boundary conditions of the spindle shaft, elastic constraints are set in the radial and thrust directions respectively to simulate the constraints of the radial and thrust bearings on the shaft.

\subsection{Fluid-thermal-solid coupling analysis of hydrostatic spindle}

Using the fluid module, thermal module and structural module in Workbench software, the fluid-thermal-solid coupling analysis of the hydrostatic spindle can be realized. In section 3.3, the temperature films of the radial bearing oil film and thrust bearing oil film with different spindle speeds under the viscosity temperature effect are obtained. The temperature fields of the radial oil film and the thrust oil film under the viscosity-temperature effect are respectively applied as loads to the hydrostatic radial bearing and the thrust bearing bush, which can obtain the temperature map of the bearing shell under the viscosity temperature effect; The temperature field of the radial oil film and the thrust oil film are applied to the spindle shaft at the same time, and the temperature and deformation clouds of the spindle shaft under the viscosity-temperature effect are obtained.

\subsection{Experimental verification}

The hydrostatic spindle experiment is conducted to verify that above simulation is valid. The test equipment consists of a hydrostatic spindle, a fuel tank, a cooling device, a loading device, and a control cabinet, as shown in Figure 3. The temperature of the radial bearing and thrust bearing of the hydrostatic bearing was detected by the Fluck-Ti32 thermal imager, and the thermal deformation of the spindle was tested by the SEA9 (Spindle Error Analyzer) rotation error analyzer produced by the American Lion Precision company, as shown in Figure 4.

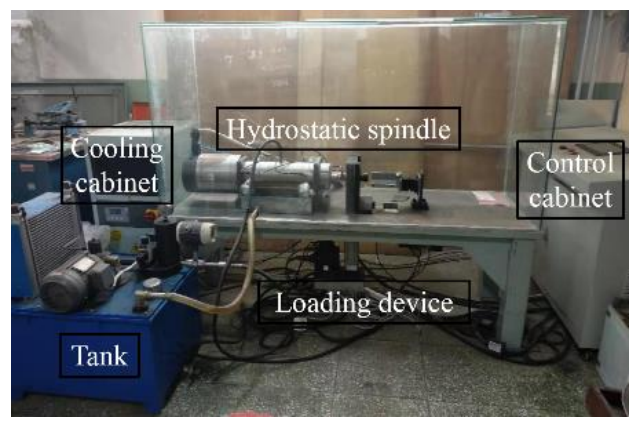

FIgure 3 Hydrostatic spindle test equipment

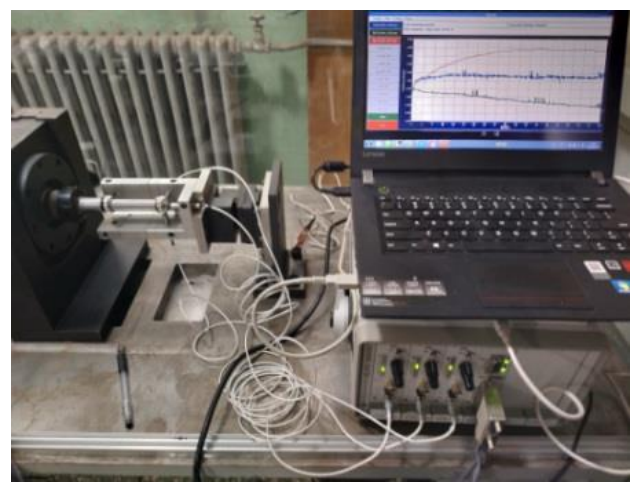

FIgure 4 Variation of temperature

\section{Results}

\subsection{Theoretical calculation results of oil film temperature} rise

\subsubsection{Theoretical calculation results}

From the formulas (1) to (14), taking into account the parameters of the radial oil film and thrust oil film, the oil film temperature rise of the radial oil film and thrust oil film are shown in the Table 2. 
Table 2 The oil film temperature rise of the radial oil film and

\begin{tabular}{cccccc}
\multicolumn{5}{c}{ thrust oil film } \\
\hline $\begin{array}{c}\text { Rotation } \\
\text { speed(rpm) }\end{array}$ & 1000 & 1200 & 1500 & 1800 & 2000 \\
\hline $\begin{array}{c}\text { Oil film } \\
\text { temperature } \\
\text { rise of the } \\
\left.\text { radial ( }{ }^{\circ} \mathrm{C}\right) \\
\begin{array}{c}\text { Oil film } \\
\text { temperature } \\
\text { rise of the } \\
\text { thrust ( }\end{array}\end{array}$ & 8.6 & 11.78 & 14.32 & 18.56 & 22.05 \\
\hline
\end{tabular}

\subsubsection{Comparative analysis of simulation and theoretical calculation of oil film temperature rise}

The difference between the oil film temperature and the inlet temperature obtained by the simulation without considering the viscosity-temperature effect and considering the viscosity-temperature effect, respectively, to obtain the temperature rise of the radial oil film and the thrust oil film when the speed is 1000rpm, 1200rpm, 1500rpm, 1800rpm, and 2000rpm , Compare and analyze the oil film temperature rise obtained by simulation and theoretical calculation, as shown in Figure 5.

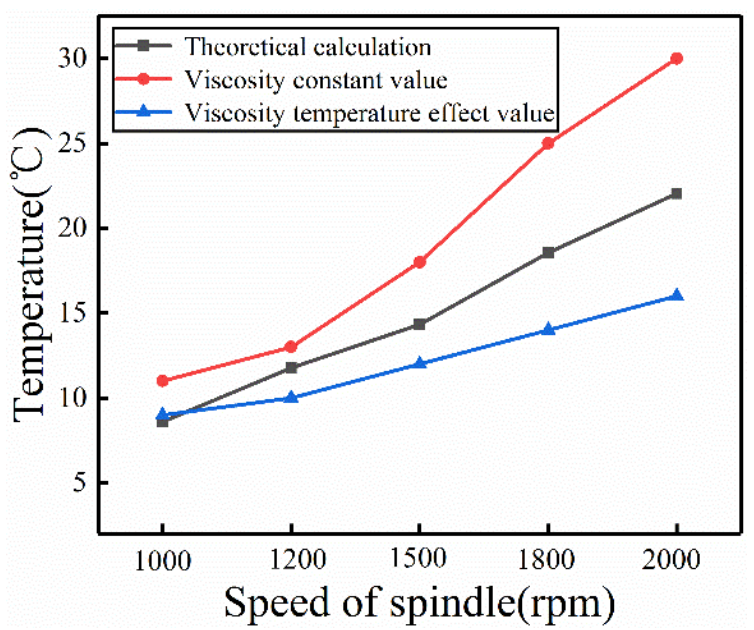

a

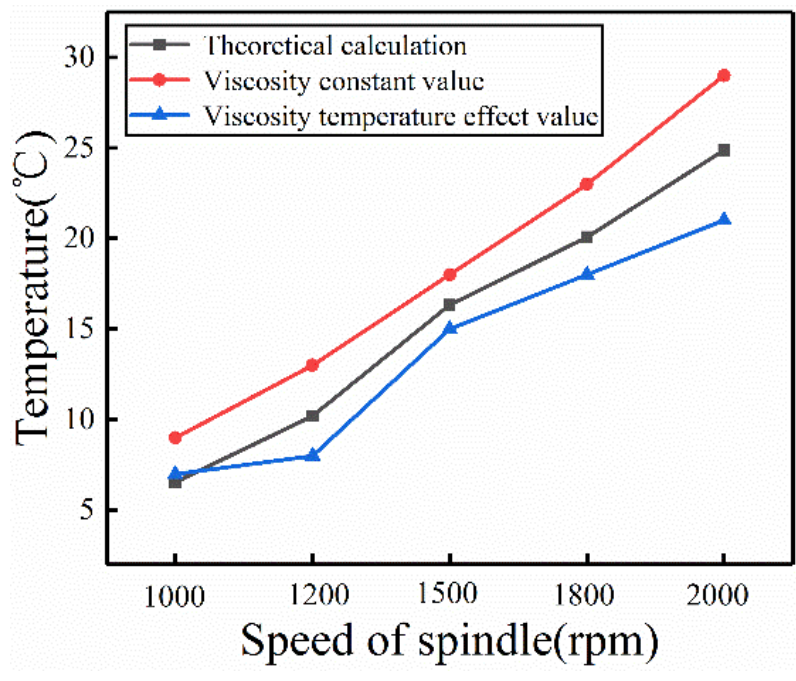

b

FIgure 5 Oil film temperature comparison: (a) Radial oil film temperature, (b) Thrust oil film temperature

\subsection{Oil film temperature cloud diagrams of hydrostatic bearing}

Based on the boundary conditions set in the previous section, the oil film temperatures are simulated at 1000rpm, 1200rpm, 1500rpm, 1800rpm, and 2000rpm, and the corresponding oil film temperatures are compared and analyzed when considering the viscositytemperature effect. The radial temperature cloud diagrams of oil film are shown in Figure 6, and the thrust temperature cloud diagrams of oil film are shown in Figure 7.

When the rotation speed is $1000 \mathrm{rpm}, 1200 \mathrm{rpm}, 1500$ rpm, $1800 \mathrm{rpm}, 2000 \mathrm{rpm}$, the radial oil film temperature and the thrust oil film temperature are shown in the Table 3. When the viscosity-temperature effect is considered, the temperature of the radial oil film and the thrust oil film are lower than the temperature when the viscosity is a fixed value, and the viscosity-temperature effect becomes more obvious as the speed increases. 
- 6 •

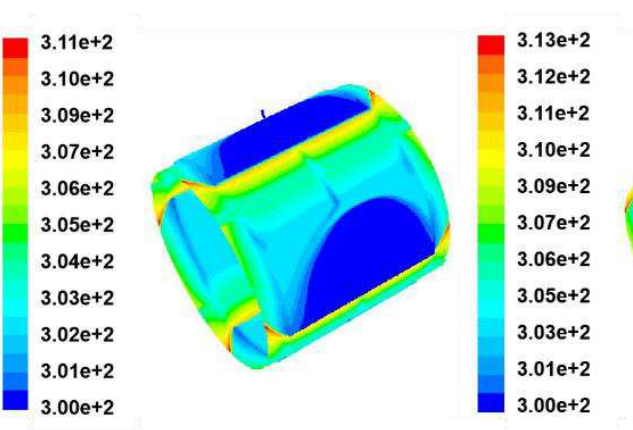

a) $n=1000 \mathrm{rpm}$

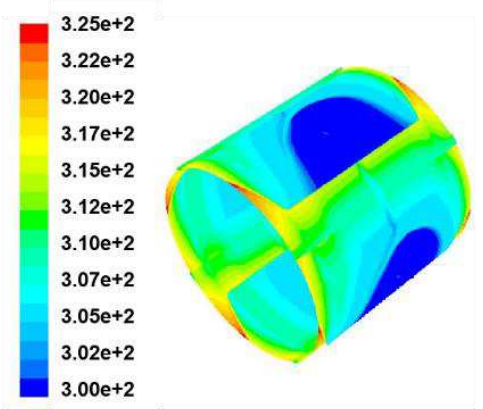

d) $n=1800 \mathrm{rpm}$
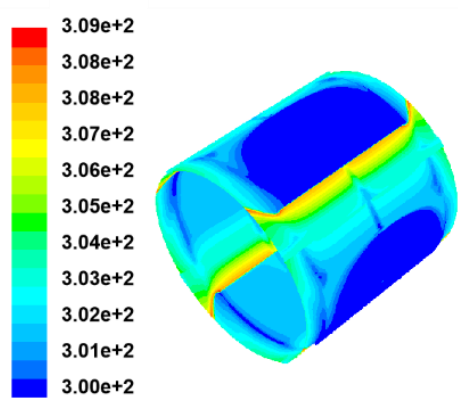

a) $\mathrm{n}=1000 \mathrm{rpm}$

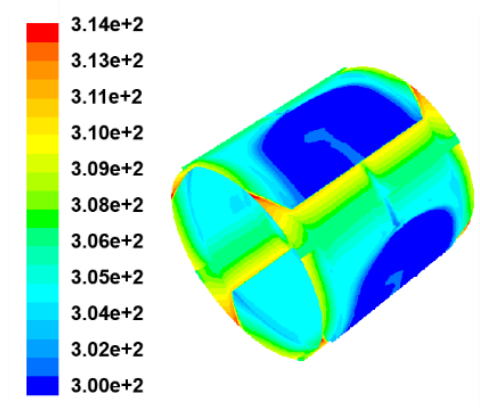

d) $\mathrm{n}=1800 \mathrm{rpm}$

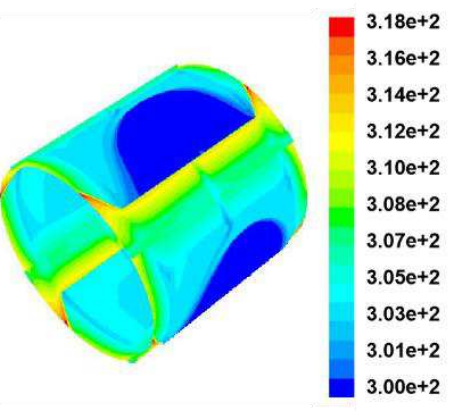

b) $\mathrm{n}=1200 \mathrm{rpm}$

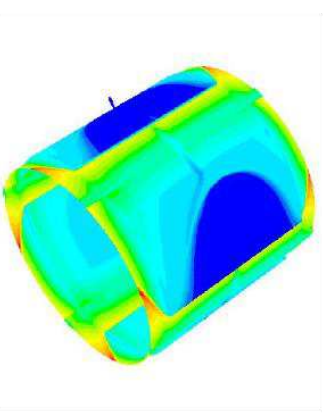

c) $\mathrm{n}=1500 \mathrm{rpm}$

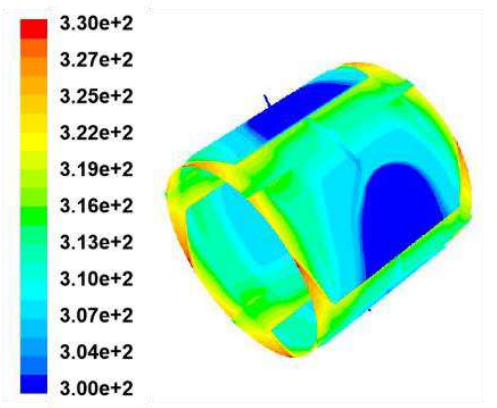

e) $n=2000 \mathrm{rpm}$

a

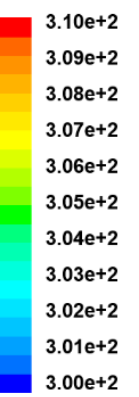

$3.00 \mathrm{e}+2$

b) $n=1200 \mathrm{rpm}$

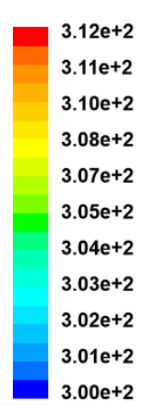

$3.16 \mathrm{e}+2$
$3.14 \mathrm{e}+2$
$3.13 \mathrm{e}+2$
$3.11 \mathrm{e}+2$
$3.09 \mathrm{e}+2$
$3.08 \mathrm{e}+2$
$3.06 \mathrm{e}+2$
$3.05 \mathrm{e}+2$
$3.03 \mathrm{e}+2$
$3.01 \mathrm{e}+2$
$3.00 \mathrm{e}+2$

b

FIgure 6 Cloud diagram of radial oil film temperature: (a) Radial oil film temperature cloud at constant viscosity, (b) Radial oil film temperature cloud diagram when viscosity changes 


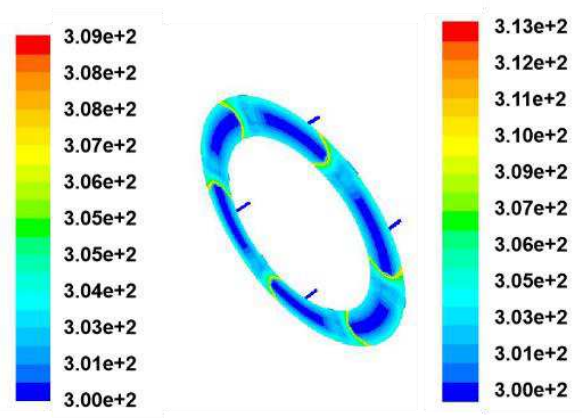

a) $\mathrm{n}=1000 \mathrm{rpm}$

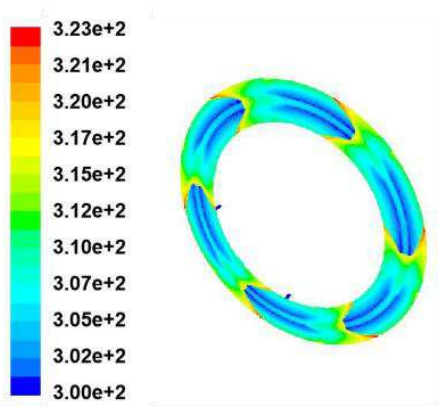

d) $\mathrm{n}=1800 \mathrm{rpm}$

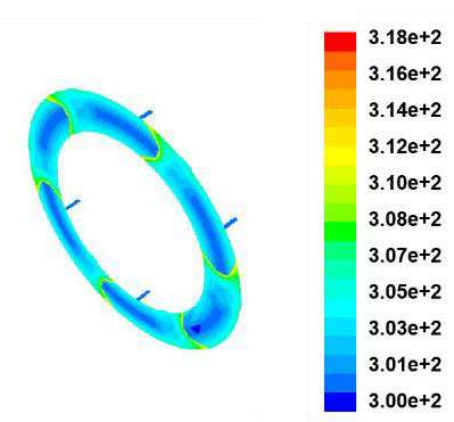

b) $\mathrm{n}=1200 \mathrm{rpm}$

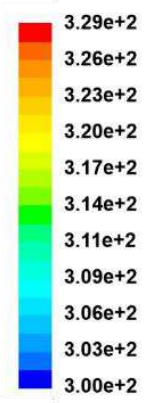

a

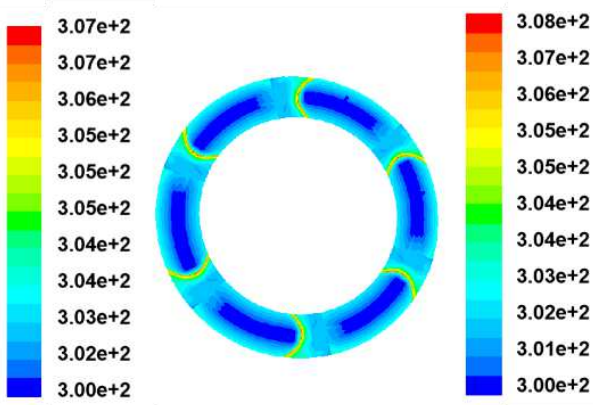

a) $n=1000 \mathrm{rpm}$

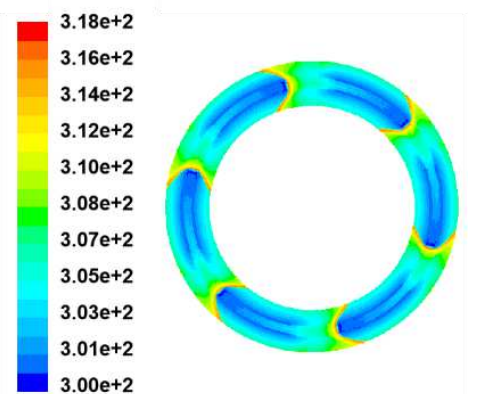

d) $n=1800 \mathrm{rpm}$

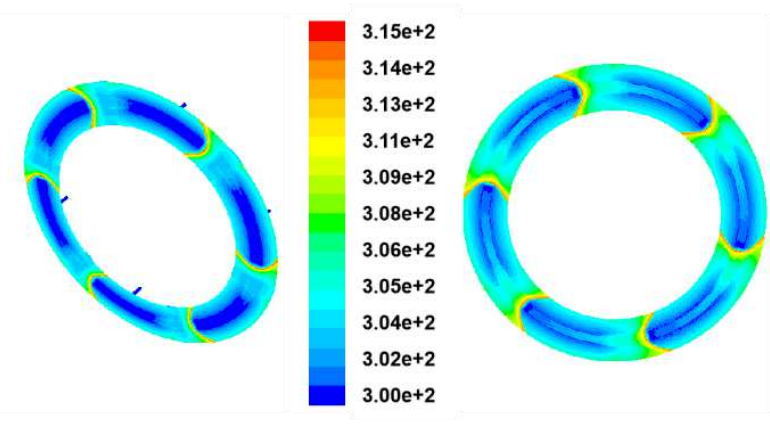

b) $n=1200 \mathrm{rpm}$

c) n=1500rpm

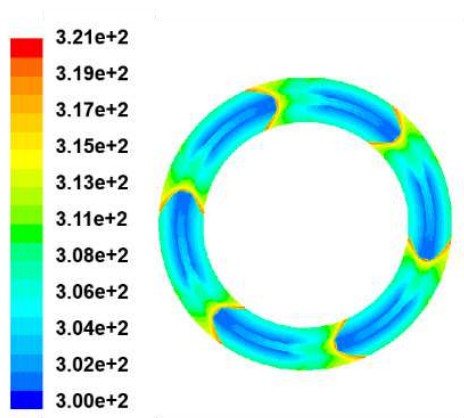

e) $n=2000 \mathrm{rpm}$

b

FIgure 7 Cloud diagram of thrust oil film temperature (a) Thrust oil film temperature cloud diagram at constant viscosity, (b) Thrust oil film temperature cloud diagram when viscosity changes 
- 8 •

Table 3 Rotation speed and oil film temperature of radial and thrust without considering viscosity-temperature effect

\begin{tabular}{cccccc}
\hline $\begin{array}{c}\text { Rotation } \\
\text { speed(rpm) }\end{array}$ & 1000 & 1200 & 1500 & 1800 & 2000 \\
\hline $\begin{array}{c}\text { radial oil film } \\
\text { temperature(k) }\end{array}$ & 311 & 313 & 318 & 325 & 330 \\
$\begin{array}{c}\text { thrust oil film } \\
\text { temperature(k) }\end{array}$ & 309 & 313 & 318 & 329 & 330
\end{tabular}

\subsection{Temperature field analysis of hydrostatic bearing}

It can be seen from Figure 8 and Figure 9, the viscositytemperature effect has a greater impact on the temperature of the radial and thrust bearings. When the viscositytemperature effect is considered, the temperature of the radial and thrust bearings is lower. As the speed increases, the viscosity-temperature effect becomes more pronounced. The higher the speed,the greater the difference in bearing temperature when the viscosity is constant and the viscosity is variabl.

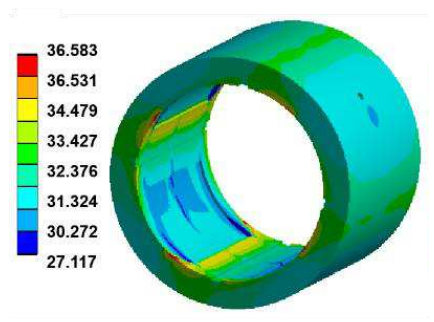

a) $\mathrm{n}=1000 \mathrm{rpm}$

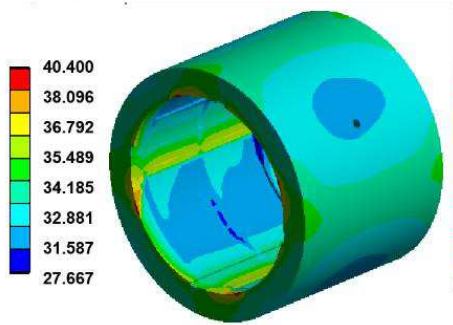

b) $\mathrm{n}=1200 \mathrm{rpm}$

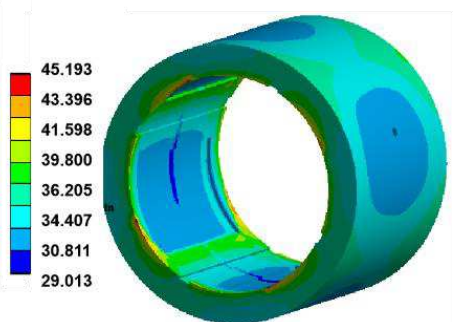

c) $\mathrm{n}=1500 \mathrm{rpm}$

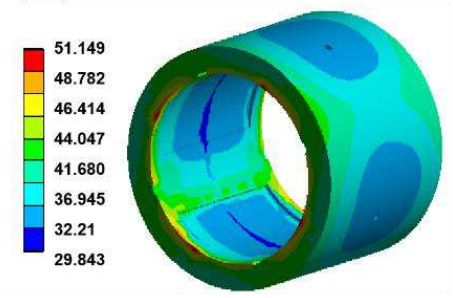

d) $\mathrm{n}=1800 \mathrm{rpm}$

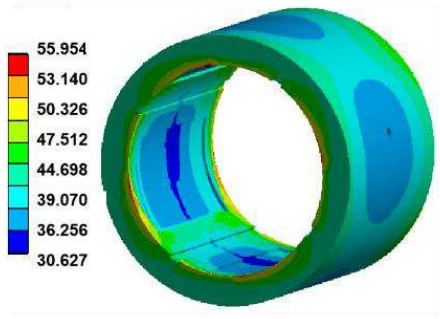

e) $n=2000 \mathrm{rpm}$

a

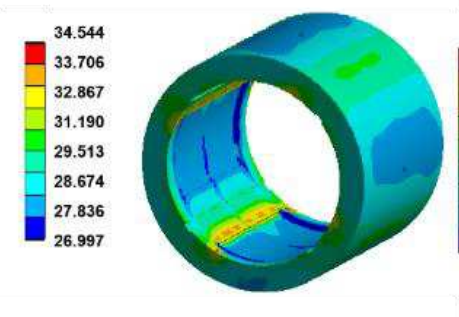

a) $n=1000 \mathrm{rpm}$

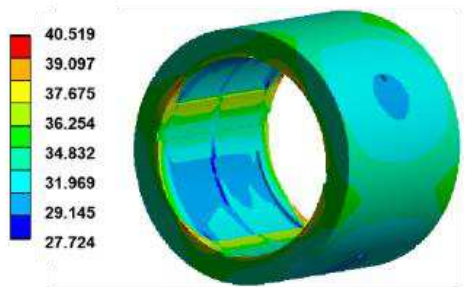

d) $\mathrm{n}=1800 \mathrm{rpm}$

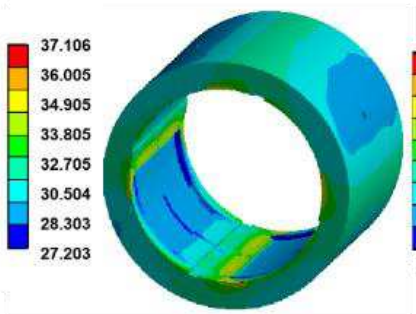

b) $\mathrm{n}=1200 \mathrm{rpm}$

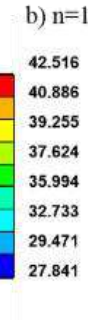

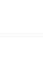

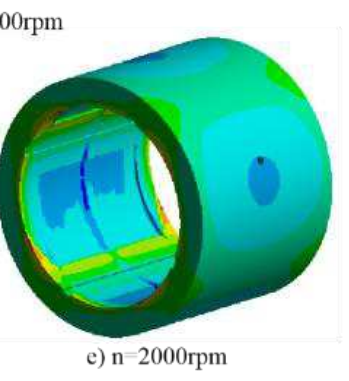

FIgure 8 Temperature cloud diagram of radial bearing pad: (a) Radial bearing bush temperature cloud diagram at constant viscosity, (b) Radial bearing bush temperature cloud diagram with viscosity change 

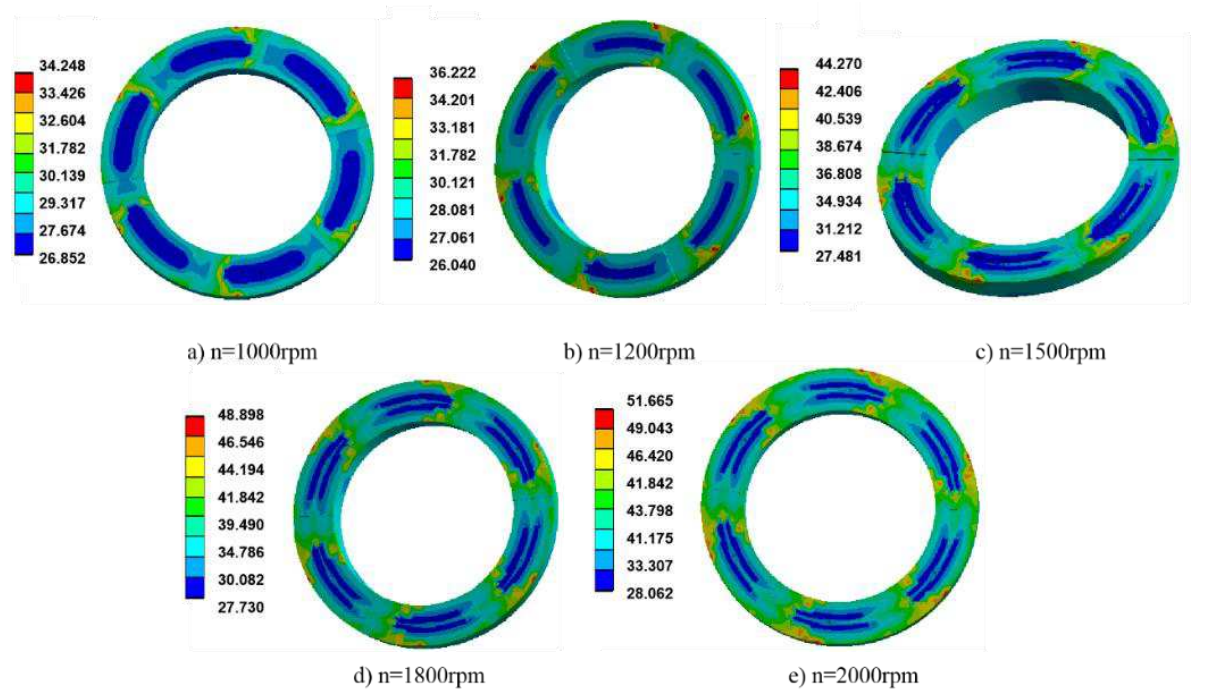

$\mathbf{a}$
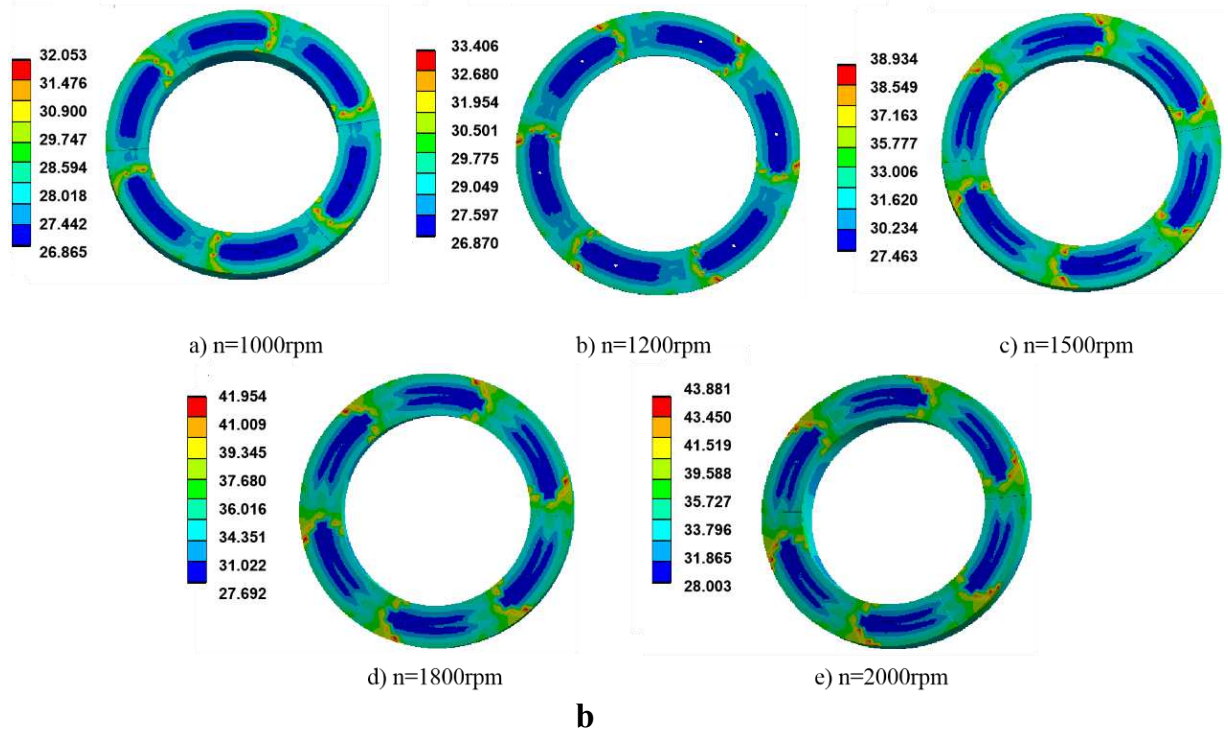

b) $\mathrm{n}=1200 \mathrm{rpm}$

c) $\mathrm{n}=1500 \mathrm{rpm}$

b

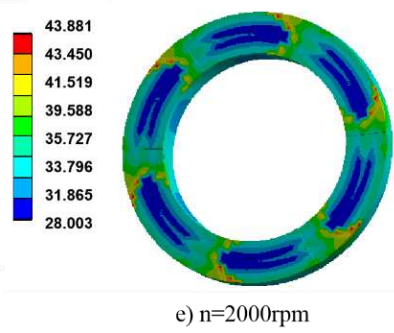

FIgure 9 Temperature map of thrust bearing bush: (a) Thrust bearing temperature cloud diagram at constant viscosity, (b) Thrust bearing temperature cloud diagram when viscosity changes

\subsection{Temperature field analysis of spindle shaft}

By coupling the temperature of the obtained radial oil film and the thrust oil film to the spindle shaft at the same time, the shaft temperature at different speeds can be obtained.As shown in Figure 10. It can be seen from Figure 10, the temperature of the spindle shaft considering the viscositytemperature effect is lower than the shaft temperature without considering the viscosity-temperature effect. 
$\cdot 10 \cdot$
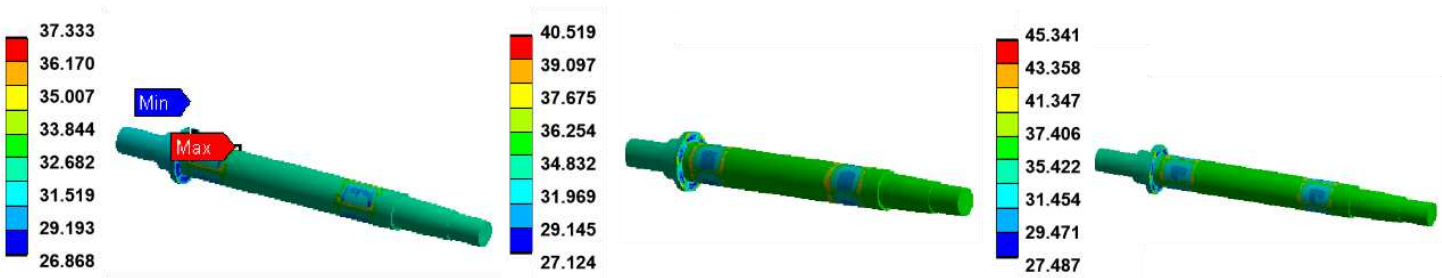

a) $\mathrm{n}=1000 \mathrm{rpm}$

b) $\mathrm{n}=1200 \mathrm{rpm}$

c) $\mathrm{n}=1500 \mathrm{rpm}$
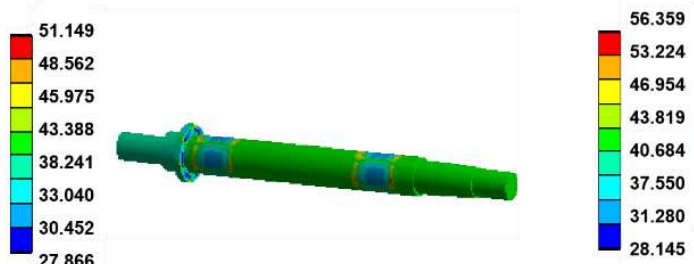

d) $n=1800 \mathrm{rpm}$

e) $n=2000 \mathrm{rpm}$

a
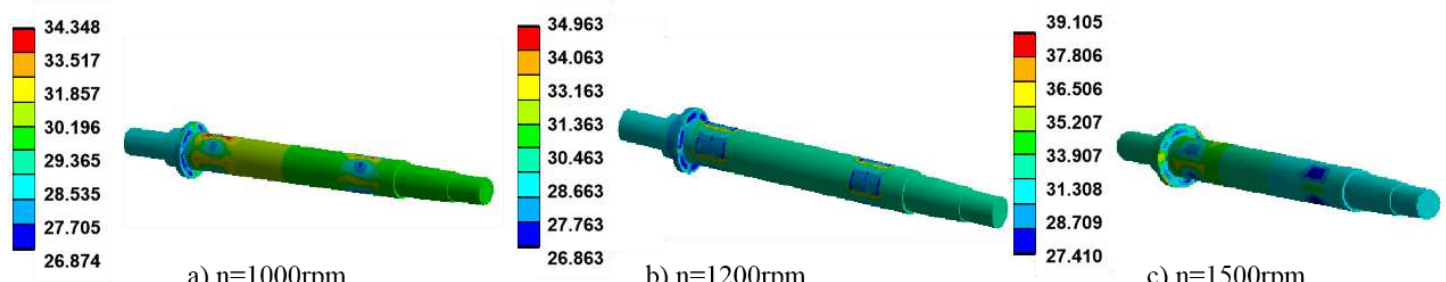

b) $\mathrm{n}=1200 \mathrm{rpm}$

c) $\mathrm{n}=1500 \mathrm{rpm}$

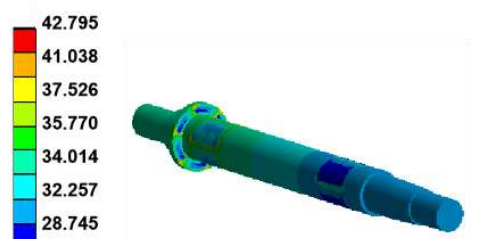

d) $n=1800 \mathrm{rpm}$

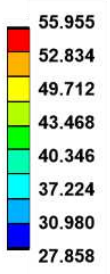

b

FIgure 10 Temperature cloud diagram of the hydrostatic spindle: (a) Shaft temperature cloud diagram at constant viscosity, (b) Shaft temperature cloud diagram at viscosity change

\subsection{Thermal deformation analysis of spindle shaft}

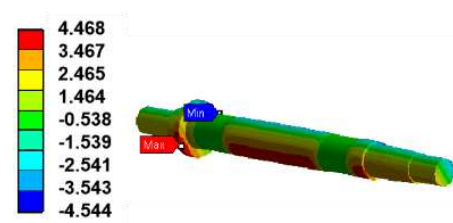

a) $\mathrm{n}=1000 \mathrm{rpm}$

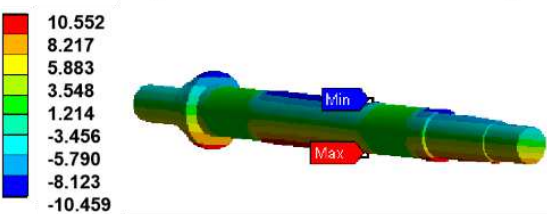

d) $\mathrm{n}=1800 \mathrm{rpm}$

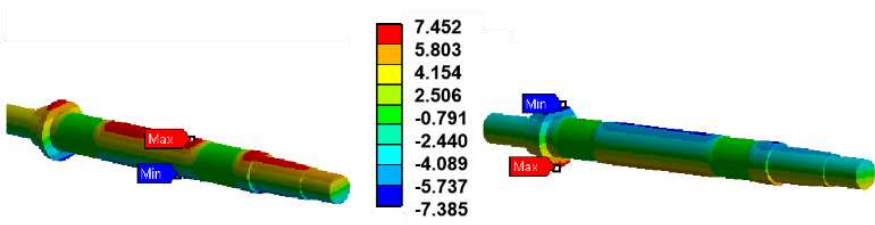

b) $n=1200 \mathrm{rpm}$

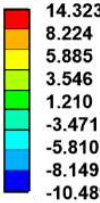

8.224
5.885
3.546
1.210
-3.471
-5.810
-8.149

$-10.489$

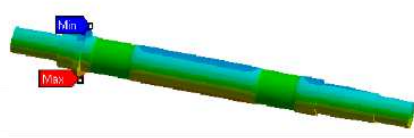

e) $n=2000 \mathrm{rpm}$ 


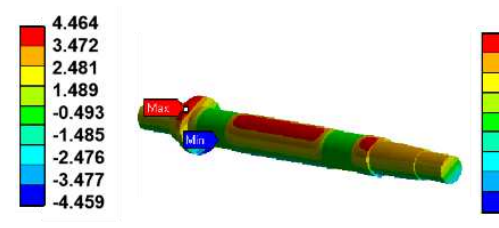

a) $\mathrm{n}=1000 \mathrm{rpm}$

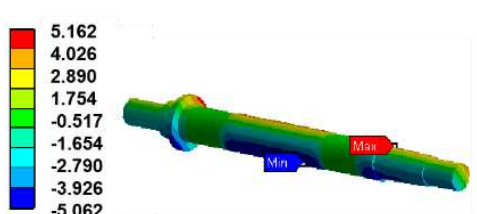

b) $n=1200 \mathrm{rpm}$

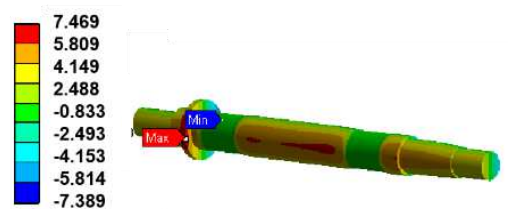

c) n=1500rpm

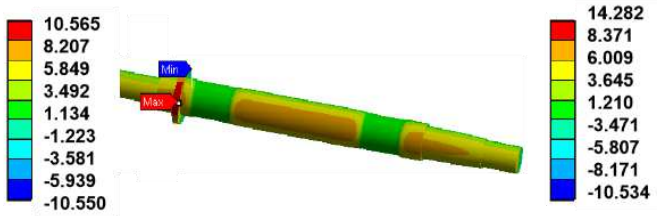

d) $n=1800 \mathrm{rpm}$

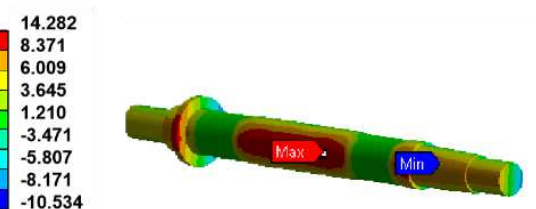

e) $n=2000 \mathrm{rpm}$

b

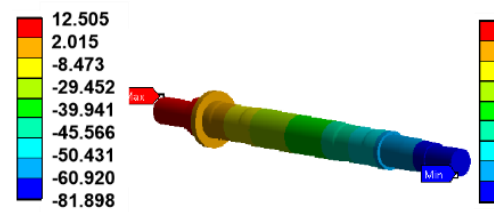

a) $n=1000 \mathrm{rpm}$

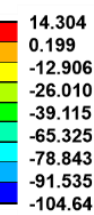

$-104.64$

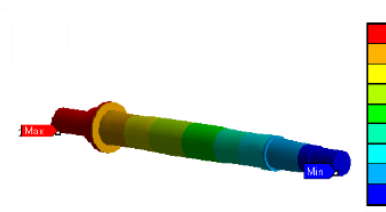

b) $n=1200 \mathrm{rpm}$

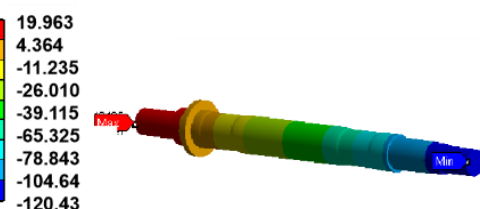

c) $\mathrm{n}=1500 \mathrm{rpm}$

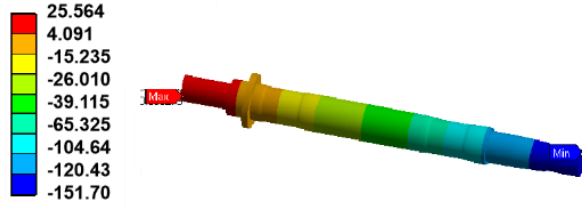

d) $\mathrm{n}=1800 \mathrm{rpm}$

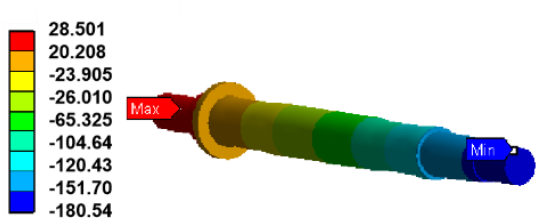

e) $n=2000 \mathrm{rpm}$

FIgure 11 Thermal deformation of spindle shaft when viscosity is fixed: (a) Shaft-X thermal deformation, (b) Shaft-Y thermal deformation, (c) Shaft-Z thermal deformation

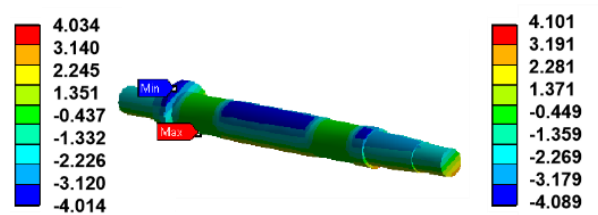

a) $\mathrm{n}=1000 \mathrm{rpm}$

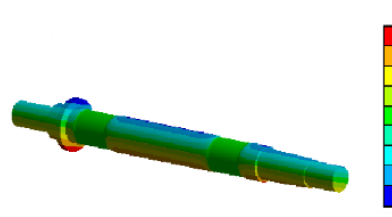

b) $n=1200 \mathrm{rpm}$

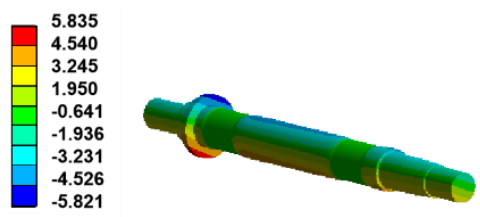

c) $\mathrm{n}=1500 \mathrm{rpm}$

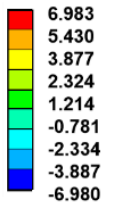

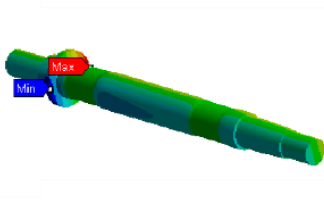

d) $n=1800 \mathrm{rpm}$

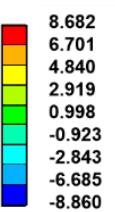

8.682
6.701
4.840
2.919
0.998
-0.923
-2.843
-6.685
-8.860

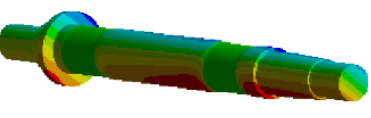

e) $n=2000 \mathrm{rpm}$

$\mathbf{a}$ 


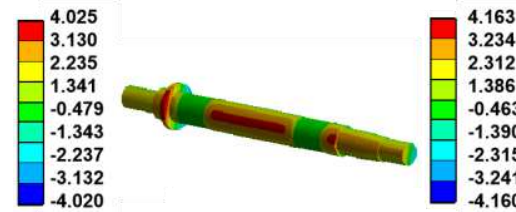

a) $\mathrm{n}=1000 \mathrm{rpm}$

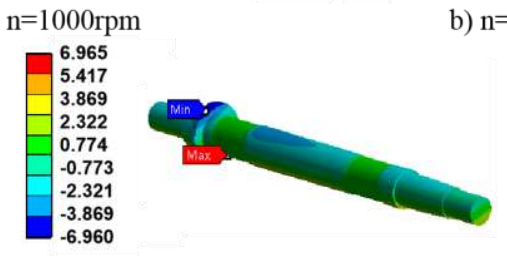

d) $n=1800 \mathrm{rpm}$

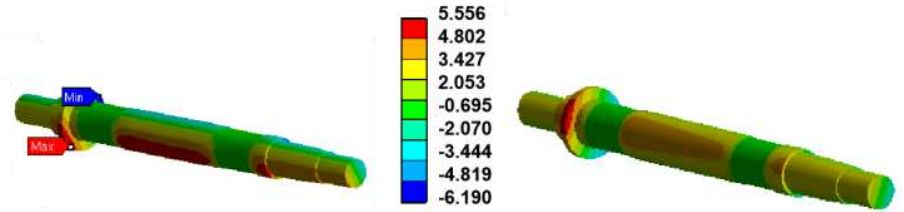

c) $n=1500 \mathrm{rpm}$

$\mathrm{n}=1200 \mathrm{rpm}$

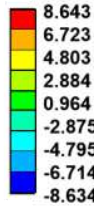

6.723

2.884

0.964
-2.875

$-4.795$

$-8.634$

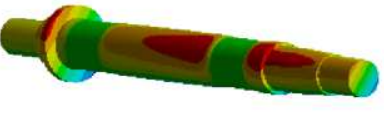

e) $n=2000 \mathrm{rpm}$

b

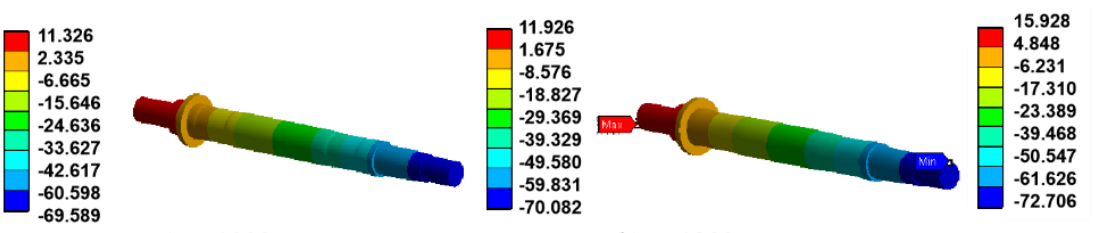

a) $\mathrm{n}=1000 \mathrm{rpm}$

b) $n=1200 \mathrm{rpm}$

c) $\mathrm{n}=1500 \mathrm{rpm}$
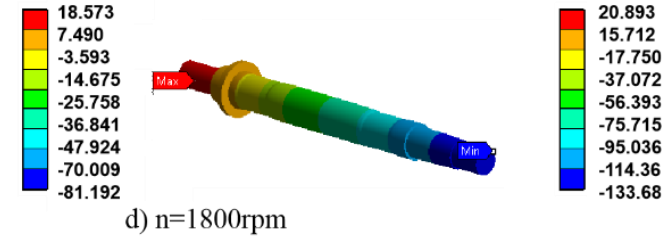

20.893
15.712

$-17.750$

3.393

$-95.036$

$-114.36$

d) $\mathrm{n}=1800 \mathrm{rpm}$

e) $n=2000 \mathrm{rpm}$

FIgure 12 Thermal deformation of spindle shaft under viscosity-temperature variation: (a) Shaft temperature cloud diagram at constant viscosity, (b) Shaft temperature cloud diagram at viscosity change

Table 4 Simulation of thermal deformation of the spindle shaft

\begin{tabular}{|c|c|c|c|c|c|c|}
\hline & & $1000 \mathrm{rpm}$ & $1200 \mathrm{rpm}$ & $1500 \mathrm{rpm}$ & $1800 \mathrm{rpm}$ & 2000rpm \\
\hline \multirow{3}{*}{$\begin{array}{l}\text { Viscosity } \\
\text { constent }\end{array}$} & $\begin{array}{l}\text { X--thermal } \\
\text { deformation } \\
(\mu \mathrm{m})\end{array}$ & 4.47 & 4.98 & 7.45 & 10.55 & 14.32 \\
\hline & $\begin{array}{l}\text { Y--thermal } \\
\text { deformation } \\
\quad(\mu \mathrm{m})\end{array}$ & 4.46 & 5.16 & 7.46 & 10.56 & 14.28 \\
\hline & $\begin{array}{l}\text { Z--thermal } \\
\text { deformation } \\
\quad(\mu \mathrm{m})\end{array}$ & 12.51 & 14.30 & 19.96 & 25.56 & 28.50 \\
\hline \multirow{3}{*}{$\begin{array}{l}\text { Viscosity } \\
\text { change }\end{array}$} & $\begin{array}{l}\text { X--thermal } \\
\text { deformation } \\
\quad(\mu \mathrm{m})\end{array}$ & 4.03 & 4.10 & 5.84 & 6.98 & 8.68 \\
\hline & $\begin{array}{l}\text { Y--thermal } \\
\text { deformation } \\
\quad(\mu \mathrm{m})\end{array}$ & 4.03 & 4.16 & 5.56 & 6.96 & 8.64 \\
\hline & $\begin{array}{l}\text { Z--thermal } \\
\text { deformation } \\
\quad(\mu \mathrm{m})\end{array}$ & 11.33 & 11.93 & 15.93 & 18.57 & 20.89 \\
\hline
\end{tabular}

The cloud diagram of the thermal deformation of the spindle at different speeds when the viscosity is fixed is shown in Figure 11. The thermal deformation cloud diagram of the spindle under the viscosity-temperature change is shown in Figure 12. When the rotation speed is $1000 \mathrm{rpm}$, $1200 \mathrm{rpm}, 1500 \mathrm{rpm}, 1800 \mathrm{rpm}, 2000 \mathrm{rpm}$, the thermal 
deformation value of the spindle shaft is shown in Table 4 .

\subsection{Temperature measurement of hydrostatic bearing}

Before measurement, the hydrostatic spindle is preheated at the start-up state. After the spindle reaches thermal equilibrium, the hydrostatic spindle speed is adjusted to $1000 \mathrm{rpm}$. The temperature of the radial and thrust bearing of the hydrostatic bearing is detected using the Fluk-Ti32 thermal imager. During the test, measure one data per minute, a total of 30 data are measured, and then use the average value as the temperature of the bearing pad. The other speed measurement methods are the same. A comparative analysis was performed between the measured temperature value of the bearing in the experiment and the simulated bearing temperature at the viscosity-temperature effect and the fixed viscosity value, and the results are shown in Figure 13.

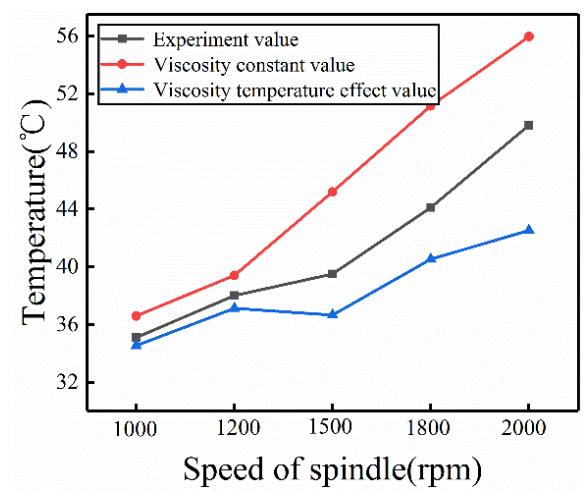

a

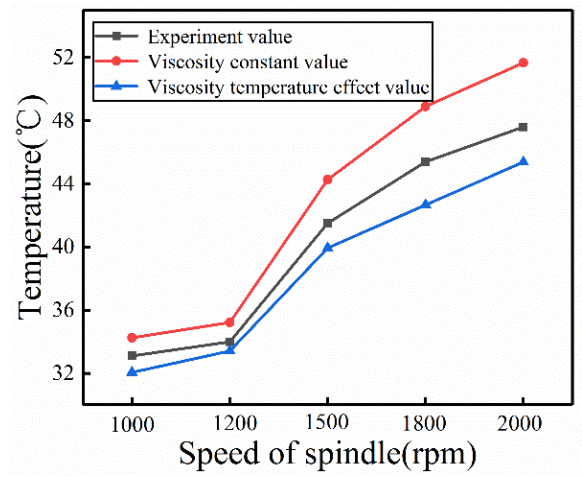

b

FIgure 13 Bearing temperature of hydrostatic bearing: (a) Radial bearing temperature, (b) Thrust bearing temperature

\subsection{Measurement of thermal deformation of hydrostatic spindle shaft}

Based on the hydrostatic spindle test bed, a SEA9 (Spindle Error Analyzer) rotary error analyzer produced by the American Lion Precision company was used to test the thermal deformation of the spindle shaft in the $\mathrm{x}, \mathrm{y}$, and $\mathrm{z}$ directions at $1000 \mathrm{rpm}, 1200 \mathrm{rpm}, 1500 \mathrm{rpm}, 1800 \mathrm{rpm}$, and $2000 \mathrm{rpm}$. After 30 minutes of test time, it tends to be in a state of thermal equilibrium. The maximum value is taken as the deformation result, and compared with the simulated viscosity constant and the thermal deformation value of the shaft under the effect of viscosity temperature. The thermal deformation of the shaft is shown in Figure 14.

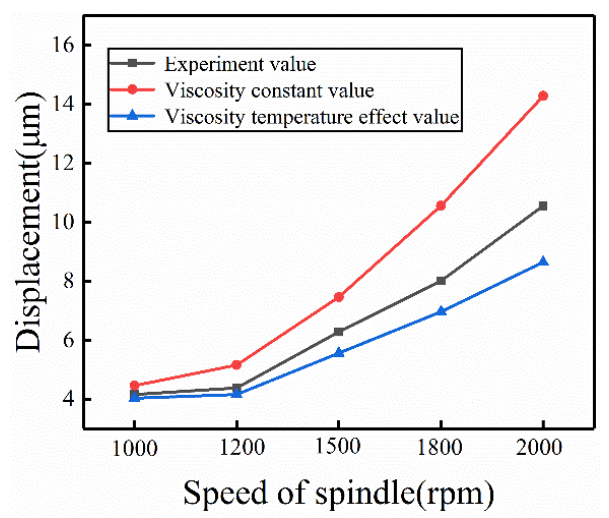

a

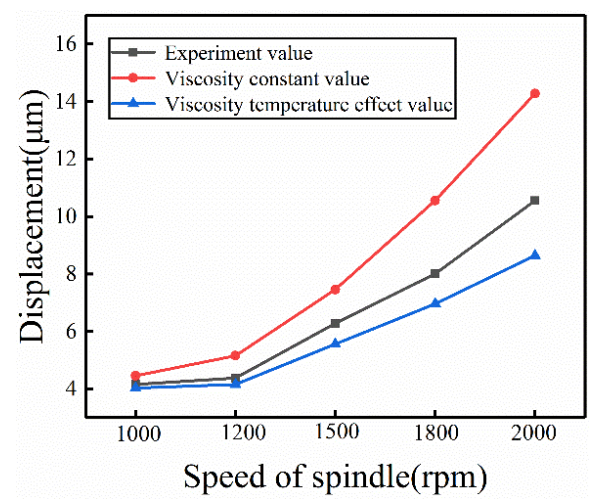

b

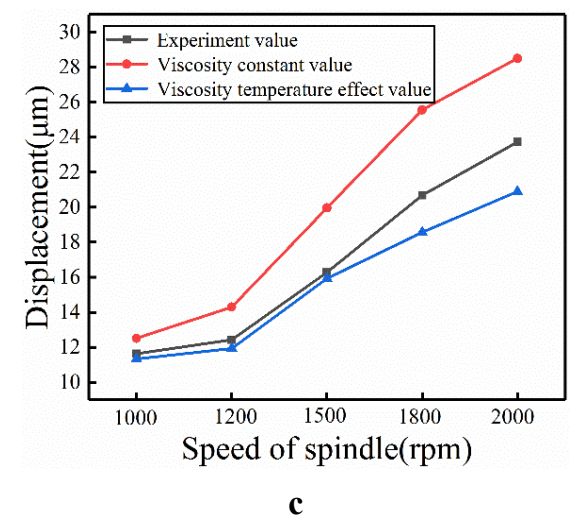

FIgure 14 Deformation of the hydrostatic spindle shaft: (a) Thermal deformation in the shaft $X$ direction, (b) Thermal deformation in the shaft $Y$ direction, (c) Thermal deformation in the $\mathrm{Z}$ direction of the shaft 


\section{Discussion}

It can be seen from Figure 5 that the oil film temperature rises significantly with the increase of the spindle speed. The simulated value of oil film temperature is compared with the theoretical value. It is found that at the same speed, the oil film temperature rise value without considering the viscosity temperature effect is greater than the theoretical calculation value, and the theoretical calculation value is greater than when the viscosity temperature effect is considered. The temperature rise of the oil film is found to be closer to the theoretical calculation value when considering the viscosity-temperature effect.

It can be concluded from Figure 6 and Figure 7 that the temperature of the radial oil film and the thrust oil film continuously increases with the increase of the rotation speed, and the high temperature region will cause a certain deflection due to the existence of the rotation speed. Due to the accumulation of lubricating oil at the oil side of the bearing shell seal, it cannot return to the oil cavity in time,the high temperature area of the radial oil film is concentrated at the positions of the radial oil seal and the axial oil seal, and the high temperature area of the thrust oil film is concentrated at the position of the circumferential oil seal. The low temperature area of the radial oil film and thrust oil film is mainly at the position of the lubricating oil inlet and the hydrostatic oil cavity, which is caused by the temperature of the lubricating oil when it enters the orifice.

It can be concluded from Figure 8 and Figure 9 that with the increase of the rotation speed, the temperatures of the radial bearing and the thrust bearing are significantly increased, and the high-temperature and low-temperature distribution positions are basically the same. The high temperature area of the radial bearing pad is distributed in the oil cavity sealing oil edge position, and the high temperature area of the thrust bearing pad is concentrated in the circumferential oil sealing position.

It can be concluded from Figure 10 that the high temperature region of the spindle shaft is distributed at the oil seal edge positions of the radial bearing and the thrust bearing, and the low temperature region is distributed at the oil cavity positions corresponding to the radial bearing and the thrust bearing. With the increase of the rotation speed, the temperature of the shaft also continues to increase.

It can be concluded from Figure 11 and Figure 12 that the thermal deformation of the spindle shaft in the axial direction is larger than the radial thermal deformation. The radial thermal deformation is almost the same in the $\mathrm{X}$ and $\mathrm{Y}$ directions. As the rotational speed increases, the spindle shaft increases continuously. When the viscositytemperature effect is considered, the thermal deformation of the shaft in three directions is smaller than the corresponding thermal deformation value without considering the viscosity-temperature effect.

It can be concluded from Figure 13 that the temperature of the radial bearing and the thrust bearing increases with the increase of the rotation speed. The value of the bearing temperature at a fixed viscosity value is greater than the experimental value, and the measured value of the experiment is greater than the value of the bearing temperature at the viscosity temperature effect. The radial bearing temperature is greater than the thrust bearing temperature at the same speed.

It can be concluded from Figure 14 that with the increase of the rotation speed, the thermal deformation of the spindle shaft in the three directions of $\mathrm{X}, \mathrm{Y}$, and $\mathrm{Z}$ increases continuously. The thermal deformation values in the $X$ and $\mathrm{Y}$ directions are basically the same. Thermal deformation measured by the experimentally is smaller than the simulated value when the specific viscosity is a fixed value, and is larger than when the viscosity-temperature effect is considered.

Based on the fluid-thermal-solid coupling, the temperature field of the hydrostatic bearing and the thermal deformation result of the shaft are obtained. The experimental value is compared with the results of the viscosity-temperature effect and the viscosity constant value, The analysis of the bearing temperature error rate is shown in Figure 15, Thermal distortion error rate analysis of the shaft in three directions is shown in Figure 16.

It can be concluded from Figure 15 that the temperature error of radial bearing and thrust bearing increases with the increase of speed, the error also becomes larger. When considering the viscosity-temperature effect, the temperature error of the radial bearing and thrust bearing is smaller than the error value when the viscosity is fixed. The temperature error rate of the bearing bush is the largest at $2000 \mathrm{rpm}$, and the temperature error rates of the radial bearing and thrust bearing are $11.05 \%$ and $7.82 \%$ when the viscosity-temperature effect is considered. The temperature error rates of the radial bearing and thrust bearing are $17.05 \%$ and $8.55 \%$ when the viscosity-temperature effect is without considered.

It can be concluded from Figure 16 that with the increase of the rotation speed, the temperature error of the spindle shaft in the three directions of $\mathrm{X}, \mathrm{Y}$, and $\mathrm{Z}$ continuously increases, reaching a maximum at $2000 \mathrm{rpm}$. The thermal distortion error is small. When considering the viscositytemperature effect, the maximum thermal deformation error rates of the shaft in the three directions of $\mathrm{X}, \mathrm{Y}$, and $\mathrm{Z}$ are $18.57 \%, 18.10 \%$, and $12.03 \%$. When the viscosity is fixed, 
the maximum thermal deformation error rates of the shaft in the three directions of $\mathrm{X}, \mathrm{Y}$, and $\mathrm{Z}$ are $34.33 \%, 35.36 \%$, and $24.4 \%$.
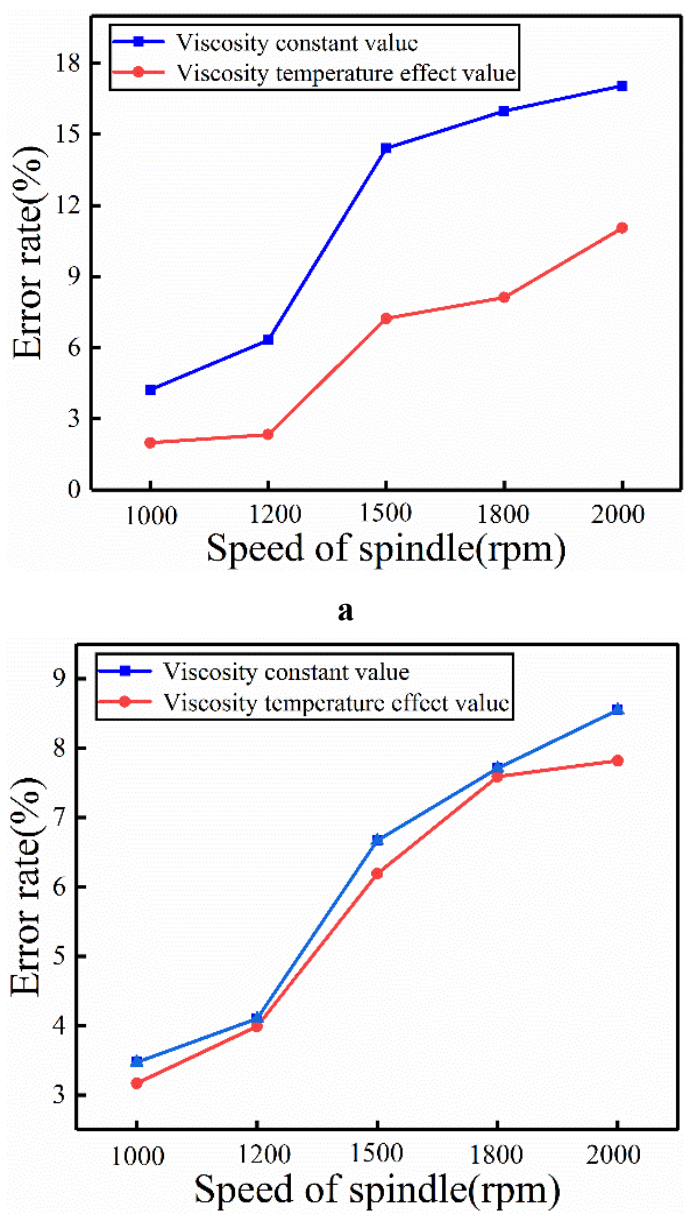

b

FIgure 15 Temperature error analysis of hydrostatic bearing bush: (a) Radial bearing temperature error, (b) Thrust bearing temperature error

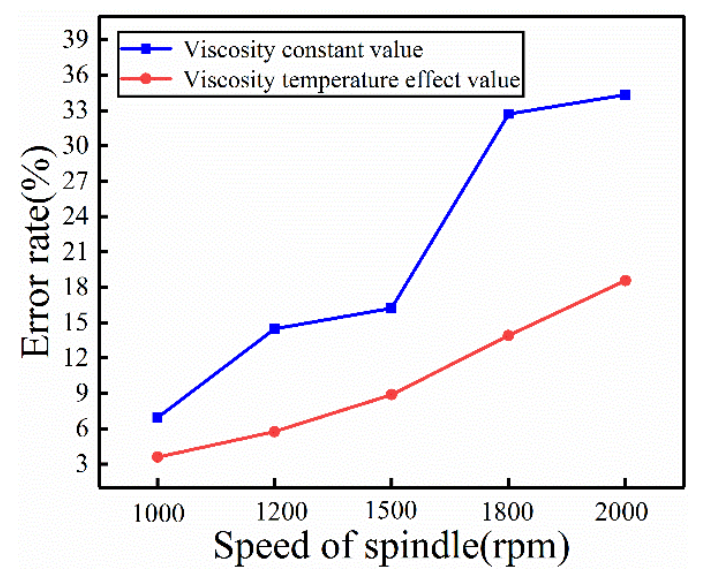

a

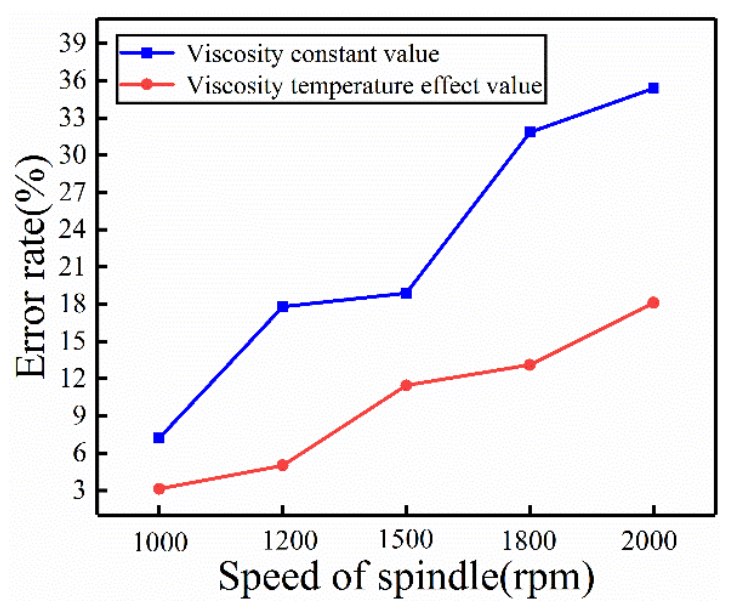

b

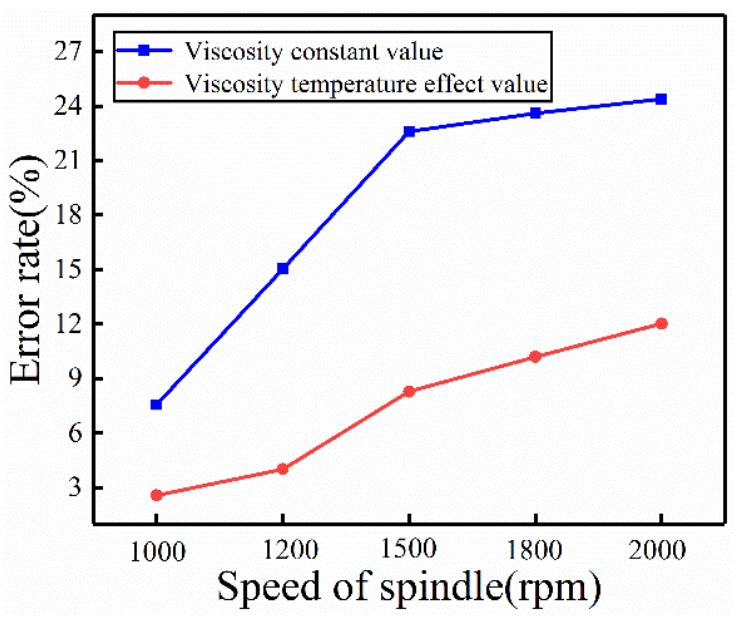

c

FIgure 16: Error analysis of temperature difference of main shaft shaft deformation, (a)Shaft X-direction thermal error, (b) Shaft Ydirection thermal error, (c) Thermal error in the $\mathrm{Z}$ direction of the shaft

\section{Conclusion}

This paper analyzes the temperature rise of the radial bearing oil film and thrust bearing oil film when the viscosity is fixed and the viscosity-temperature effect is considered. Then according to the fluid-heat-solid coupling analysis theory. The temperature field of the oil film is applied to the bearing and the shaft as a temperature load, and then the temperature cloud map of the bearing bush and the shaft and the deformation cloud map of the shaft are obtained. Finally, the simulation models are compared by experiments.

(1) According to the Newtonian shear stress formula, the temperature rise of the radial oil film and the thrust oil film at different speeds are calculated in the paper. The oil film temperature rise of the radial oil film at the speeds of 
$1000 \mathrm{rpm}, 1200 \mathrm{rpm}, 1500 \mathrm{rpm}, 1800 \mathrm{rpm}$, and $2000 \mathrm{rpm}$ is $8.60^{\circ} \mathrm{C}, 11.78^{\circ} \mathrm{C}, 14.32^{\circ} \mathrm{C}, 18.56^{\circ} \mathrm{C}, 22.05^{\circ} \mathrm{C}$. And the oil film temperature rise of the thrust oil film at the speeds of $1000 \mathrm{rpm}, 1200 \mathrm{rpm}, 1500 \mathrm{rpm}, 1800 \mathrm{rpm}$, and $2000 \mathrm{rpm}$ is $6.55^{\circ} \mathrm{C}, 10.21^{\circ} \mathrm{C}, 16.33^{\circ} \mathrm{C}, 20.08^{\circ} \mathrm{C}, 24.87^{\circ} \mathrm{C}$.

(2) The main factor influencing the temperature rise of the hydrostatic bearing oil film is the speed. With the increase of the speed, the viscosity temperature effect becomes more obvious. When the viscosity temperature effect is considered at $2000 \mathrm{rpm}$ and when the viscosity temperature effect is not considered, the radial oil film temperature difference reaches $14^{\circ} \mathrm{C}$ and the thrust oil film temperature difference also reached $8{ }^{\circ} \mathrm{C}$. The oil film temperature rise when the viscosity-temperature effect is considered is closer to the theoretical calculation value.

(3) Considering the viscosity-temperature effect and the constant viscosity value, it is found that the thermal deformation of the spindle shaft in the axial direction is more obvious than in the radial direction. When the viscosity is fixed, the axial thermal deformation is almost twice that of the radial thermal deformation. When considering the viscosity-temperature effect, the axial deformation exceeds the radial deformation by 2 times.

(4) The larger the speed, the greater the error rate of the temperature of the hydrostatic spindle bearing bush and the thermal deformation of the shaft. When considering the viscosity-temperature effect, the error rate of the bearing temperature and the thermal deformation of the shaft is smaller than the error rate when the viscosity is fixed. Considering the effect of viscosity and temperature, the maximum error rates of the thermal deformation of the shaft in the axial and radial directions are $12.03 \%$ and $18.57 \%$. The temperature error rates of the radial bearing and the thrust bearing are $11.05 \%$ and $7.82 \%$. When the viscosity is fixed, the maximum error rates of the thermal deformation of the shaft in the axial and radial directions are $24.4 \%$ and $35.36 \%$. The temperature error rates of the radial bearing and the thrust bearing are $17.05 \%$ and $8.55 \%$.

\section{Acknowledgements}

Not applicable

\section{Authors'Contributions}

$\mathrm{XZ}$ has carried on the simulation and the experiment, and has compiled the manuscript. DC was in charge of the whole project and directed the studies. ALL authors read and approved the final manuscript.

\section{Authors'Information}

Dongju Chen, born in 1980,is currently an professor at Beijing University of Technology, China. He received his $\mathrm{PhD}$ degree from
Harbin Institute of Technology, China, in 2010. His research interests include Precision and ultra precision machining.

Xuan Zhang, born in 1999, is currently a master candidate at CNC precision machining Laboratory, Beijing University of Technology, China.

\section{Funding}

This research was funded by the National Natural Science Foundation of China Grant No. (51875005), National Science and Technology Major Project of China (2016ZX04003001).

\section{Competing Interests}

The authors declare no competing financial interest.

Availability of data and materials

The datasets supporting the conclusions of this article are included within the article.

Author Details

Mechanical Industry Key Laboratory of Heavy Machine Tool Digital Design and Testing, Faculty of Materials and Manufacturing, Beijing University of Technology, Beijing 100124, China

\section{References}

[1] S Ibaraki, $\mathrm{H}$ Inui, $\mathrm{C}$ Hong, et al. On-machine identification of rotary axis location errors under thermal influence by spindle rotation. Precision Engineering, 2019, 55: 2-47.

[2] D J Chen, M Bonis, F H Zhang, S Dong. Thermal error of a hydrostatic spindle. Precision Engineering, 2011, 35: 512-520.

[3] J L Liu, C Ma, S L Wang, et al. Thermal-structure interaction characteristics of a high-speed spindle bearing system. International Journal of Machine Tools and Manufacture, 2019, 137:42-57.

[4] L Lu, H Su, Y Liang, et al. Research on Static Stiffness of Hydrostatic Bearing using Fluid-Structure Interaction Analysis. rocedia Engineering, 2012, 29(4):1304-1308.

[5] M B Yu, X D Yu, X H Zheng, H Jiang. Thermal-fluid-solid coupling deformation of hydrostatic thrust bearing friction pairs. Industrial Lubrication and Tribology, 2019, 71(3).

[6] F Tan, L Wang, M Yin, et al. Obtaining more accurate convective heat transfer coefficients in thermal analysis of spindle using surrogate assisted differential evolution method. Applied Thermal Engineering, 2019, 149:1335-1344.

[7] Y F Zhang, T Liu, W G Gao, Y L Tian. Active coolant strategy for thermal balance control of motorized spindle unit. Applied Thermal Engineering, 2018, 134:460-468.

[8] X Z Wang, T B Yu, Y H Song, et al . Thermal characteristics analysis of liquid hybrid bearing on ultra-high speed grinding. Advanced Materials Research, 2012, 565: 171-176 .

[9] J P Shao, G Liu, X Yu. Simulation and experiment on pressure field characteristics of hydrostatic hydrodynamic hybrid thrust bearings[J]. Industrial Lubrication and Tribology, 2019, 71(1).

[10] S Xiang, X Yao, Z C Du, J G Yang. Dynamic linearization modeling approach for spindle thermal errors of machine tools[J]. Mechatronics. 2018, 53: 215-228.

[11] $\mathrm{K}$ Yan, J Hong, J H Zhang, W Mi. Thermal-deformation coupling in thermal network for transient analysis of spindlebearing system[J]. International Journal of Thermal Sciences. 2016, 104: 1-12. 
[12] T Holkup, $\mathrm{H}$ Cao, et al. Thermo-mechanical model of spindles. CIRP Annals-Manufacturing Technology, 2010, 59(1): 365-368.

[13] H Su, LH Lu, Y C Liang, Q Zhang, Y Z Sun. Thermal analysis of the hydrostatic spindle system by the finite volume element method. Int J Adv Manuf Technol, 2014, 71: 1949-1959.

[14] Q Y Meng, X X Yan, C C Sun, L Yang. Research on thermal resistance network modeling of motorized spindle based on the influence of various fractal parameters. International Communications in Heat and Mass Transfer, 2020, 117:104806.
[15] C Y Wu, S T Xiang, W S Xiang. Spindle thermal error prediction approach based on thermal infrared images: A deep learning method. Journal of Manufacturing System, 2021, 59: 67-80.

[16] J L Liu, C Ma, H Q Gui, S L Wang. Thermally-induced error compensation of spindle system based on long short term memory neural networks. Applied Soft Computing Journal, 2021, 102:107094. 
Figures

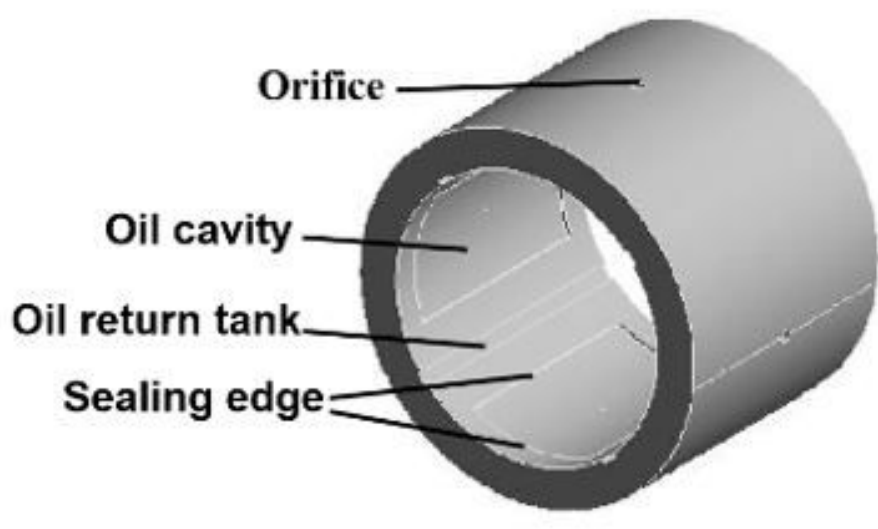

a

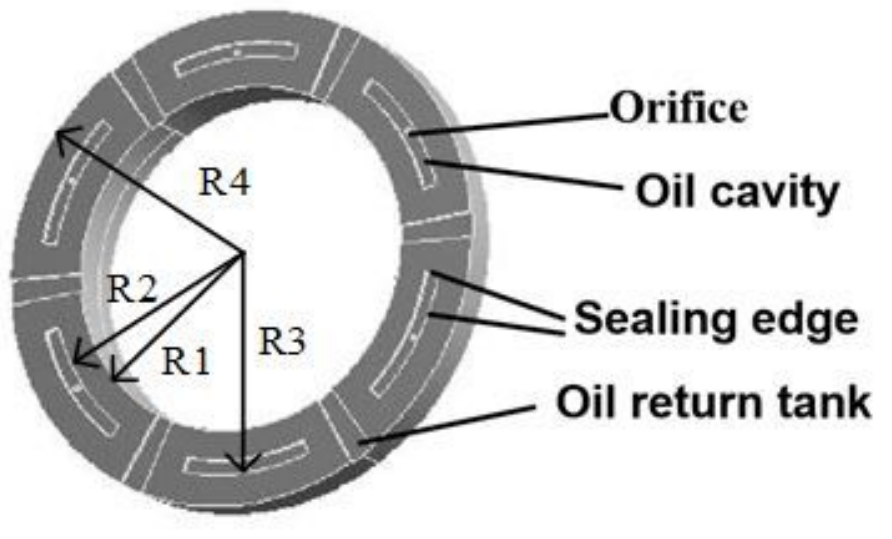

b

Figure 1

Structure of hydrostatic bearing: (a) Radial bearing, (b) Thrust bearing

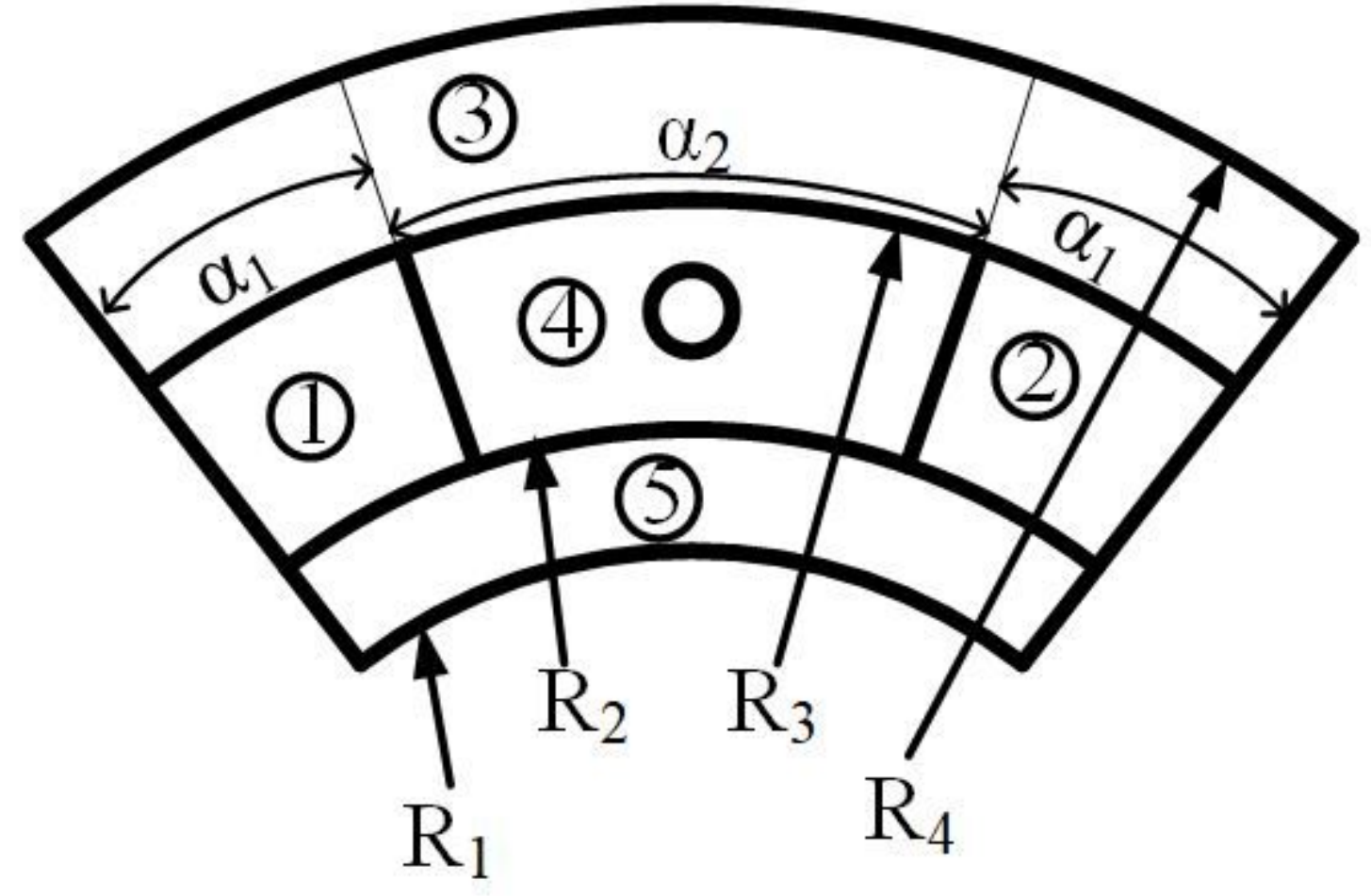

Figure 2 
Simplified model of thrust bearing seal surface

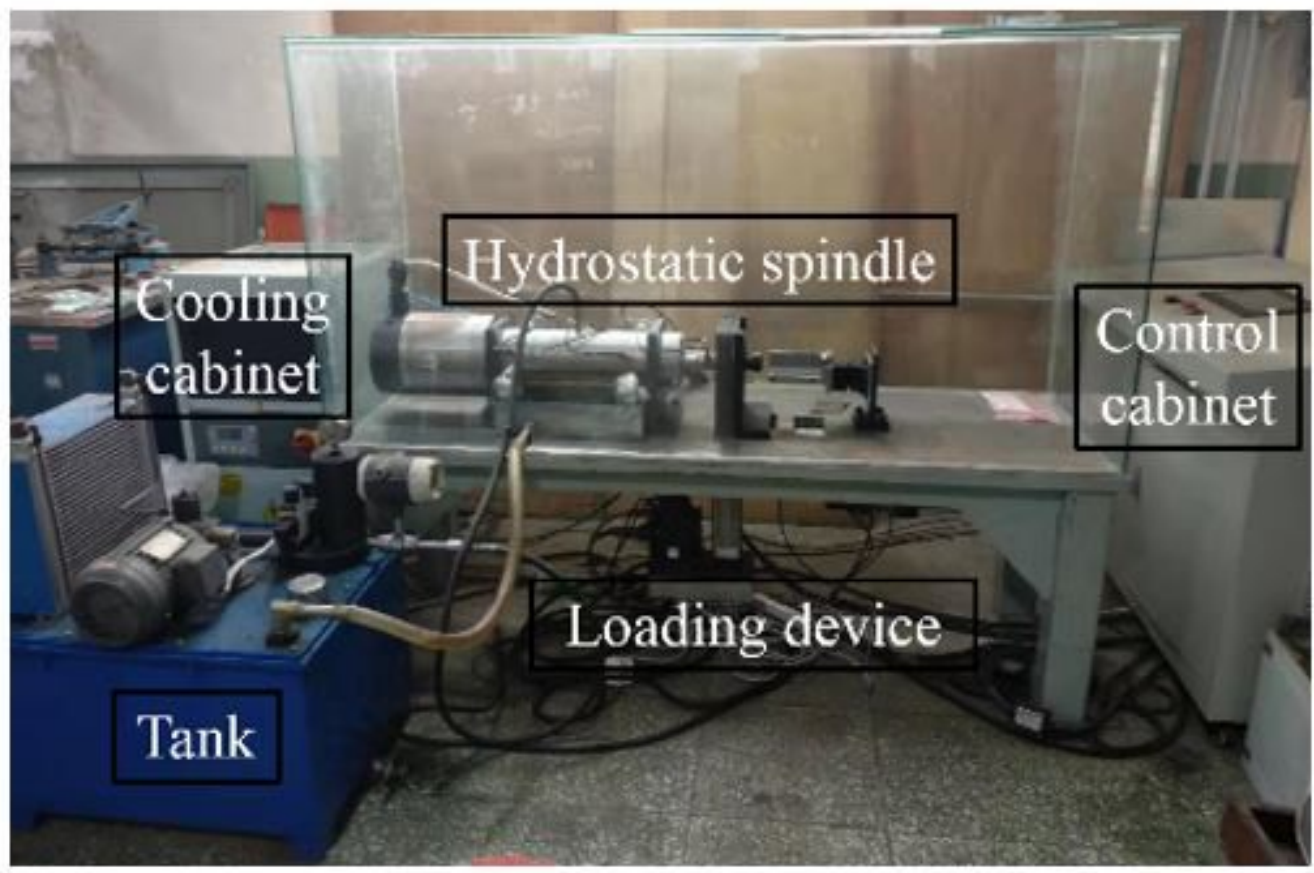

\section{Figure 3}

Hydrostatic spindle test equipment

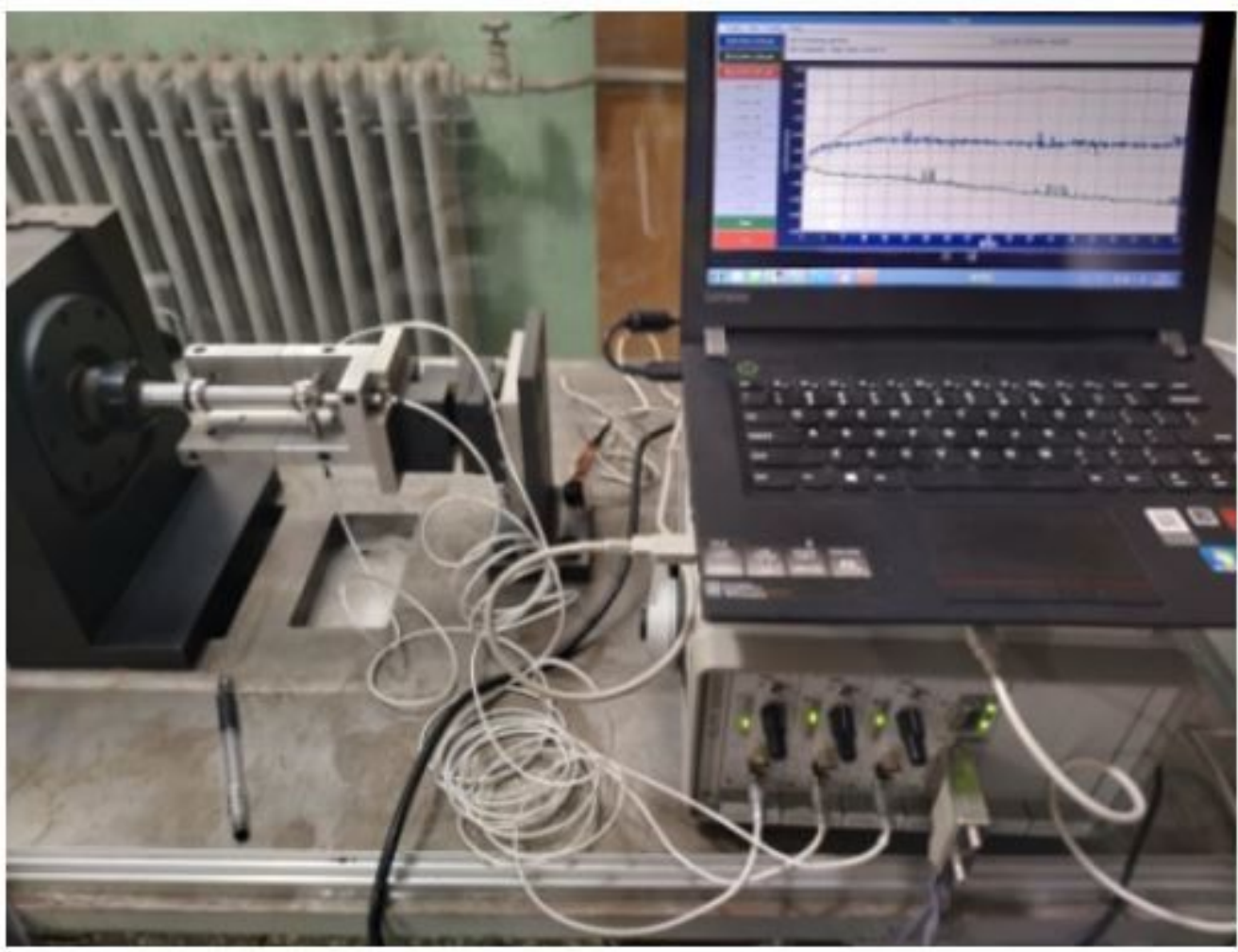

Figure 4 


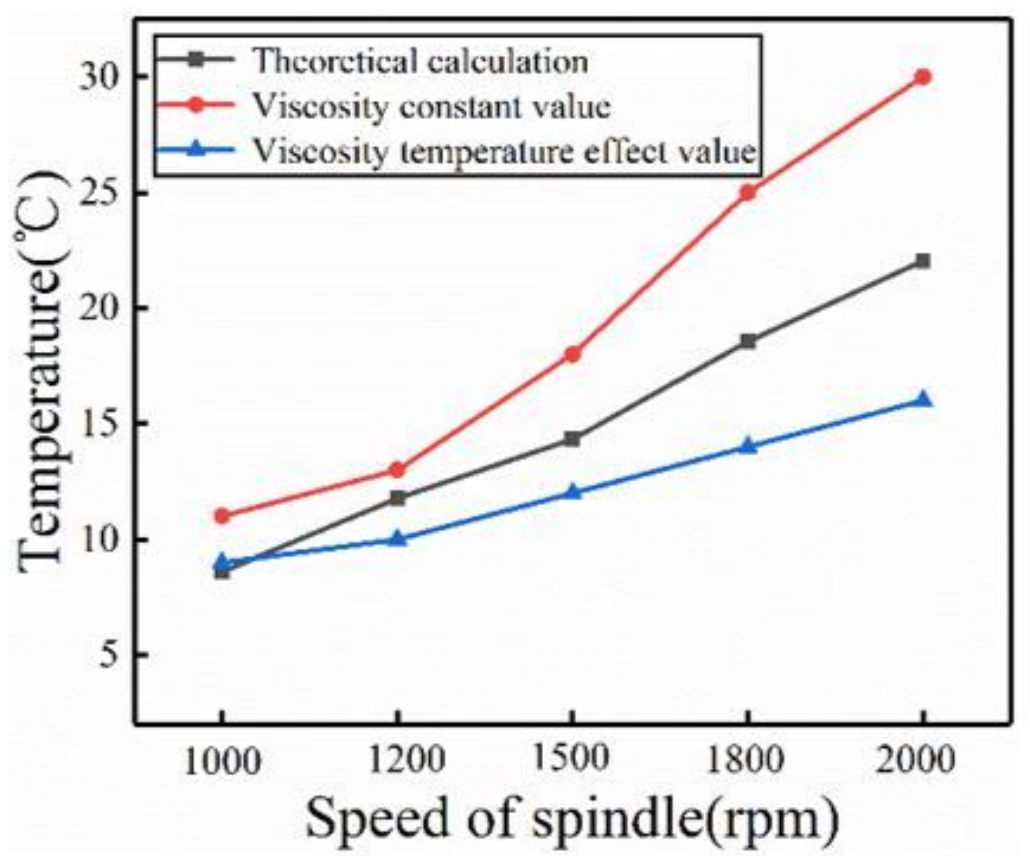

a

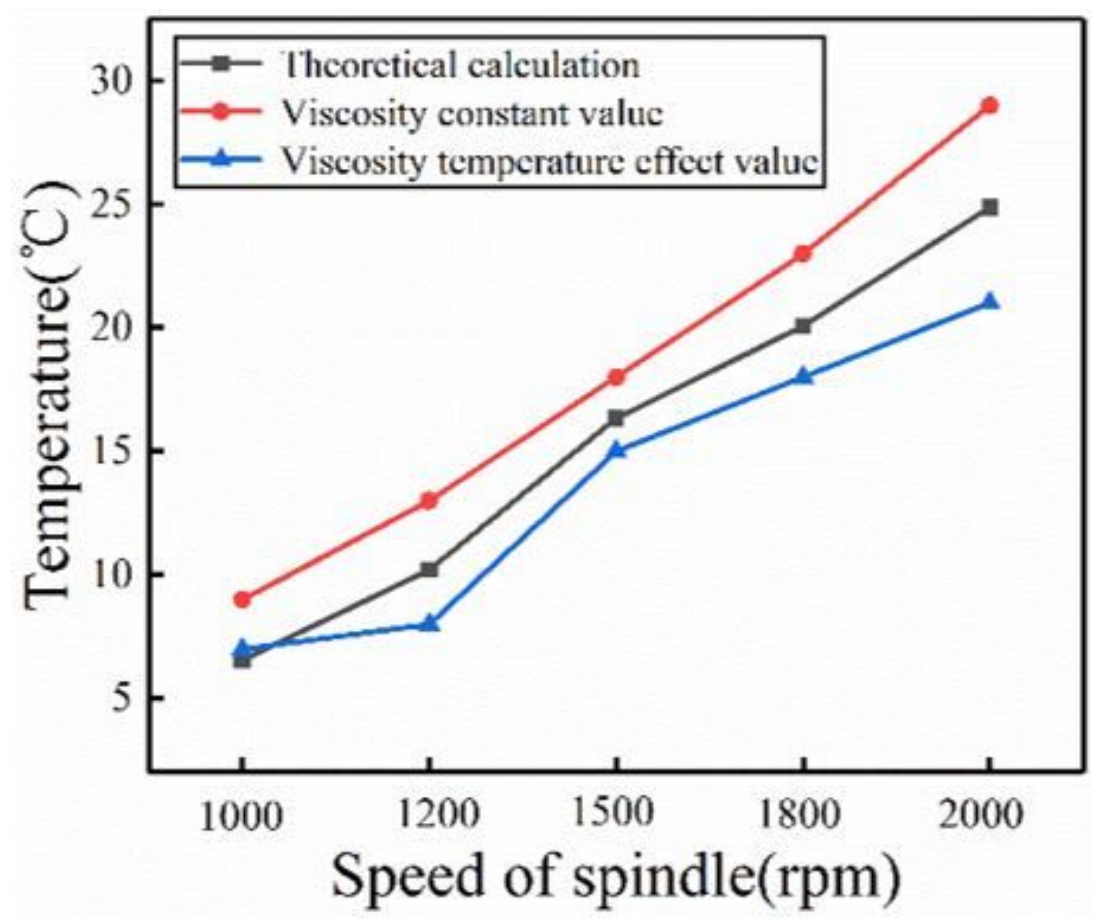

b

\section{Figure 5}

Oil film temperature comparison: (a) Radial oil film temperature, (b) Thrust oil film temperature 


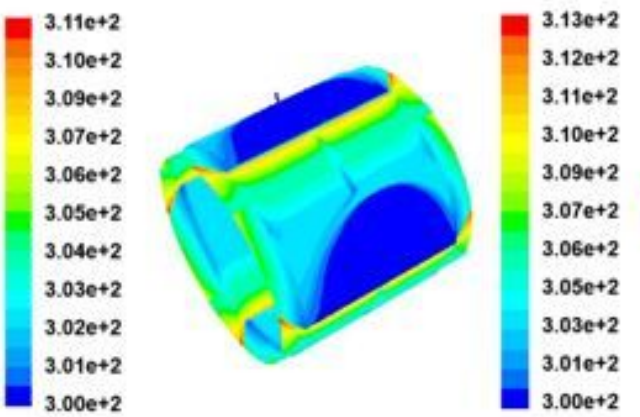

a) $\mathrm{n}=1000 \mathrm{rpm}$

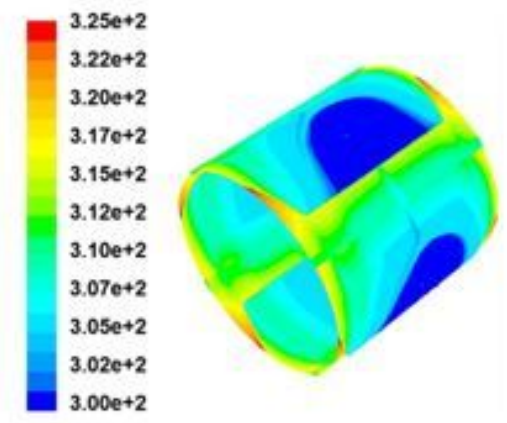

d) $\mathrm{n}=1800 \mathrm{rpm}$
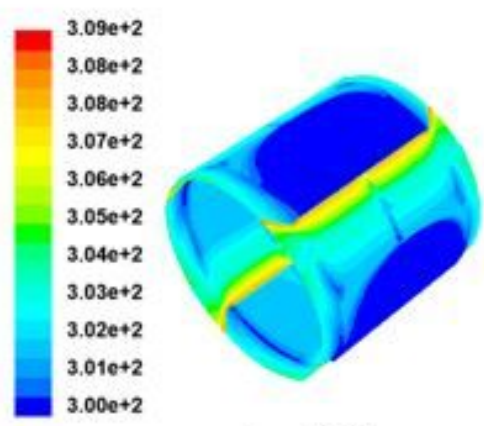

a) $n=1000 \mathrm{rpm}$

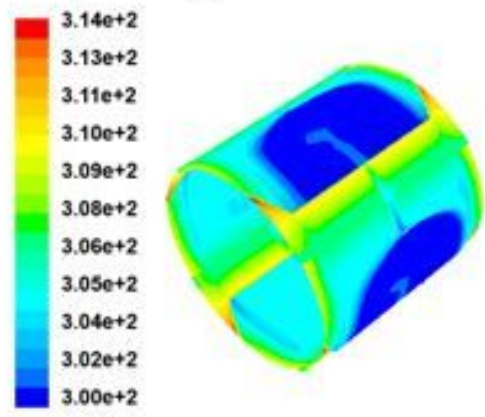

d) $\mathrm{n}=1800 \mathrm{rpm}$

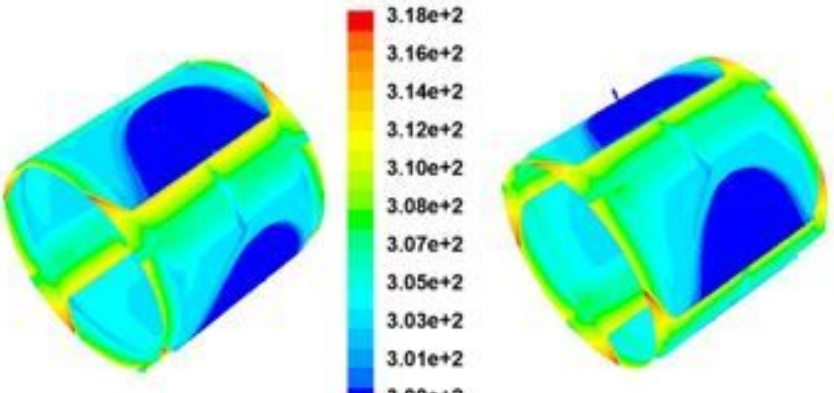

b) $\mathrm{n}=1200 \mathrm{rpm}$

c) $\mathrm{n}=1500 \mathrm{rpm}$

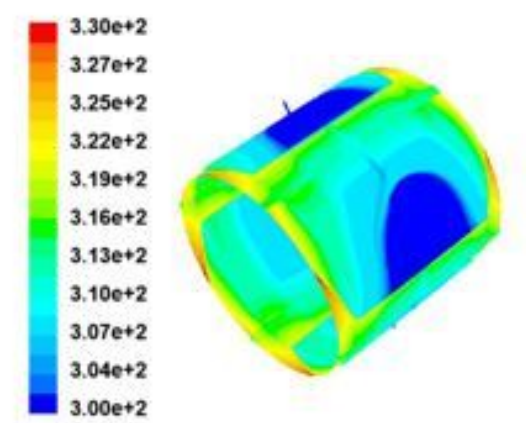

e) $n=2000 \mathrm{rpm}$

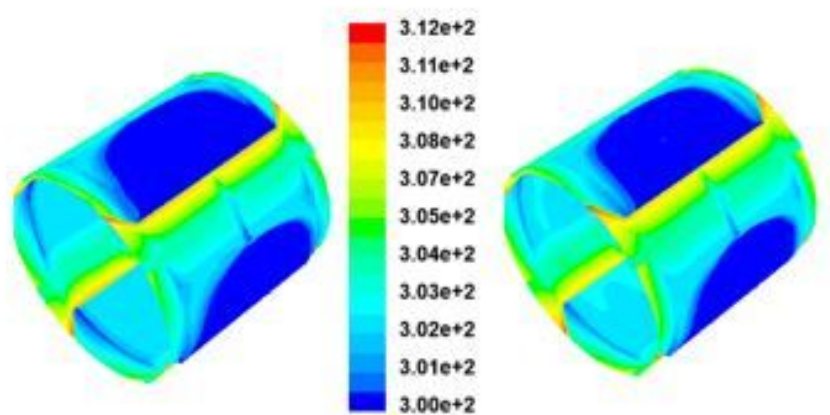

b) $\mathrm{n}=1200 \mathrm{rpm}$

c) $\mathrm{n}=1500 \mathrm{rpm}$

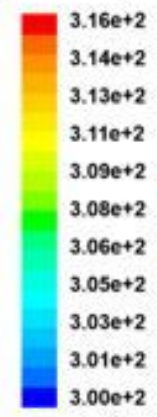

b

\section{Figure 6}

Cloud diagram of radial oil film temperature: (a) Radial oil film temperature cloud at constant viscosity, (b) Radial oil film temperature cloud diagram when viscosity changes 


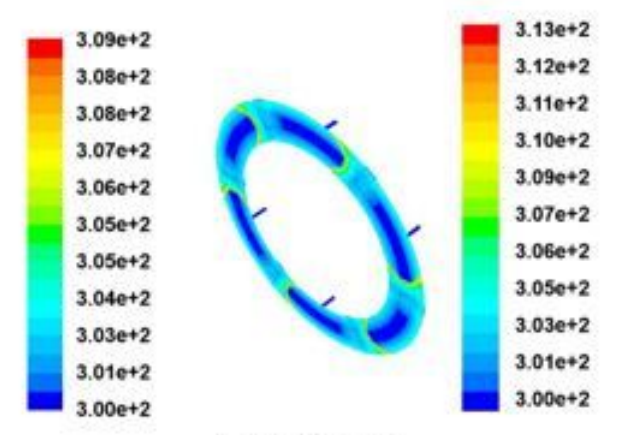

a) $\mathrm{n}=1000 \mathrm{rpm}$

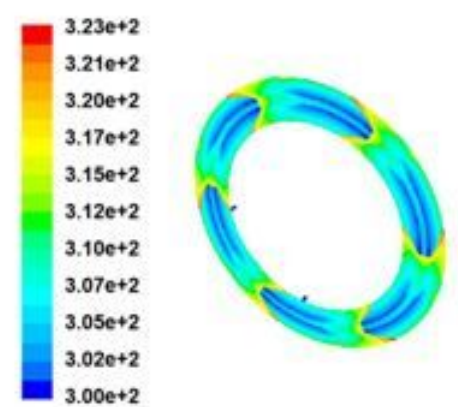

d) $n=1800 \mathrm{rpm}$

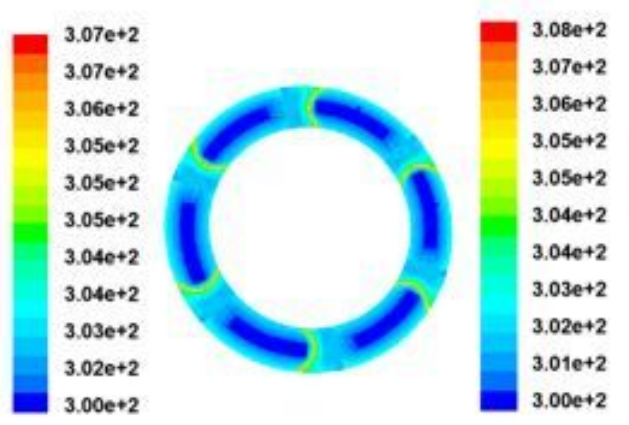

a) $\mathrm{n}=1000 \mathrm{rpm}$

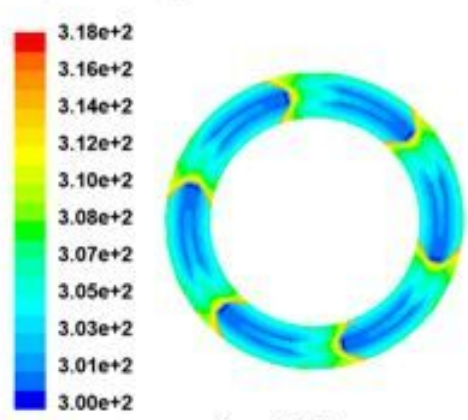

d) $\mathrm{n}=1800 \mathrm{rpm}$
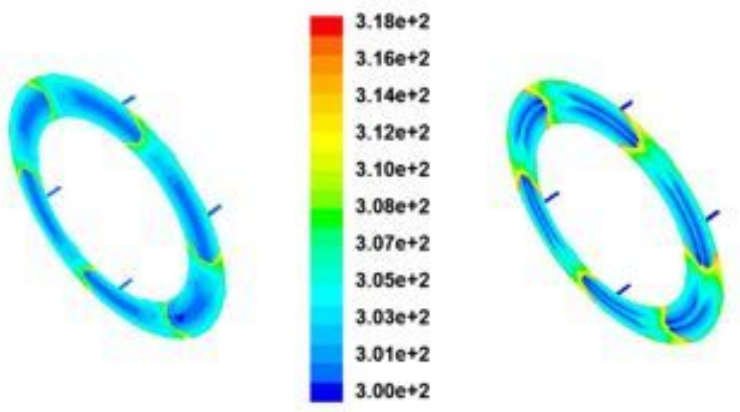

b) $\mathrm{n}=1200 \mathrm{rpm}$

c) $\mathrm{n}=1500 \mathrm{rpm}$

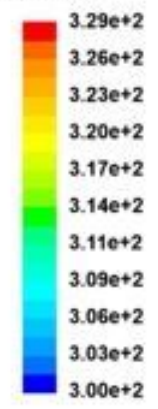

$3.26 \mathrm{e}+2$

$3.23 \mathrm{e}+2$

$3.20 e+2$

$3.17 e+2$

. $06 \mathrm{e}+2$

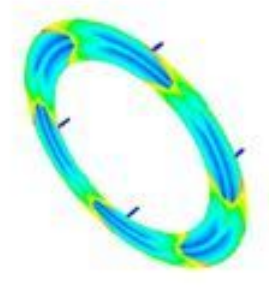

e) $n=2000 \mathrm{rpm}$

a

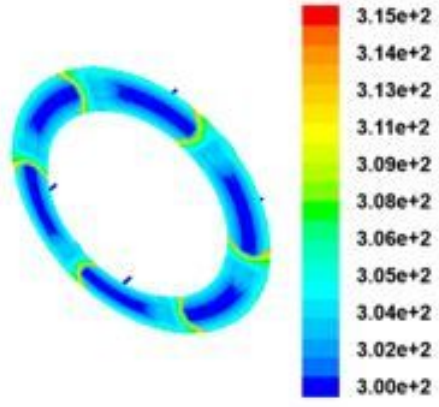

b) $\mathrm{n}=1200 \mathrm{rpm}$

c) $\mathrm{n}=1500 \mathrm{rpm}$

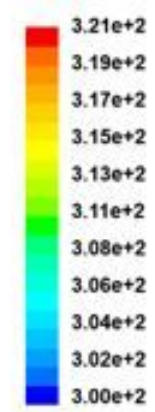

b

\section{Figure 7}

Cloud diagram of thrust oil film temperature (a) Thrust oil film temperature cloud diagram at constant viscosity, (b) Thrust oil film temperature cloud diagram when viscosity changes 


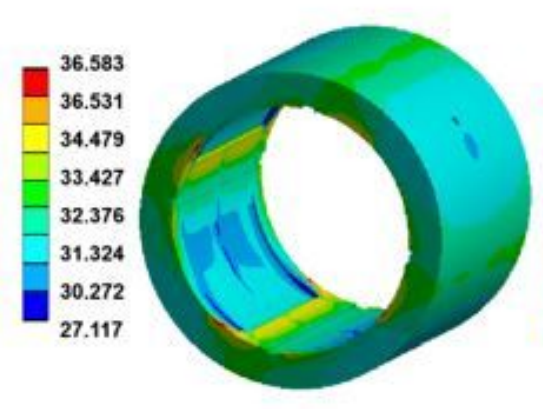

a) $\mathrm{n}=1000 \mathrm{rpm}$

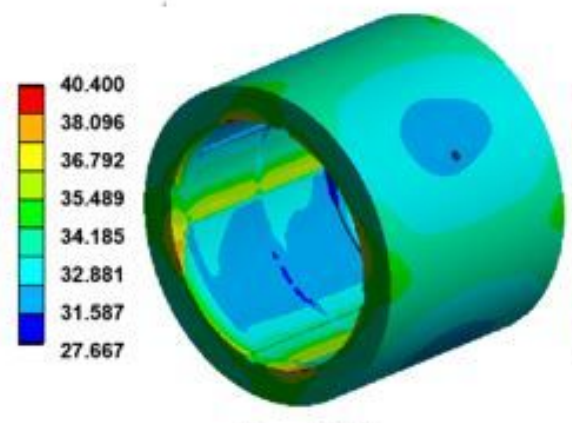

b) $n=1200 \mathrm{rpm}$
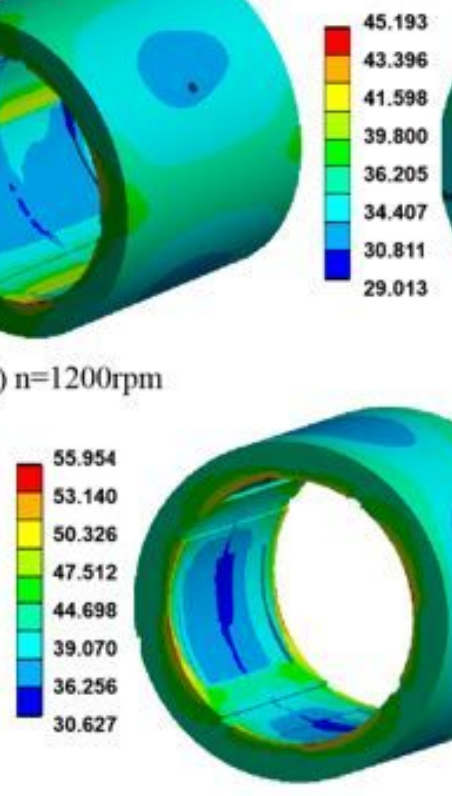

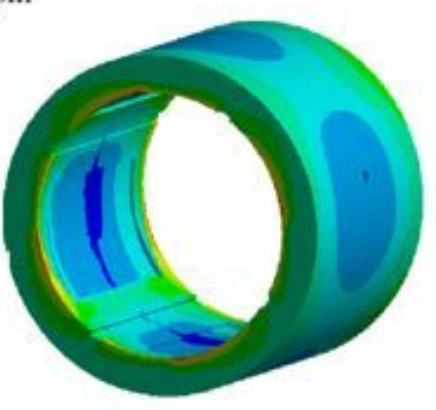

e) $n=2000 \mathrm{rpm}$

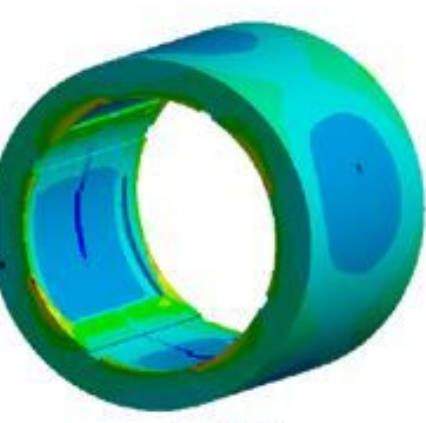

c) $\mathrm{n}=1500 \mathrm{rpm}$ d) $n=1800 \mathrm{rpm}$

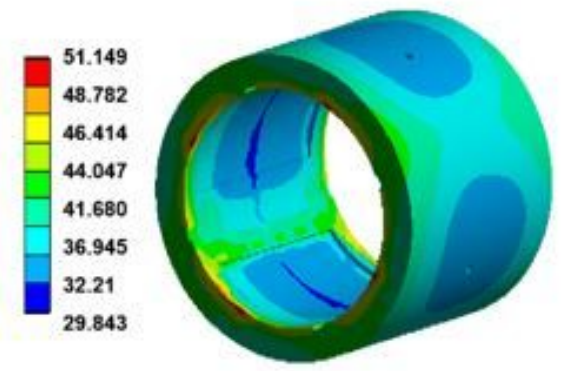

a

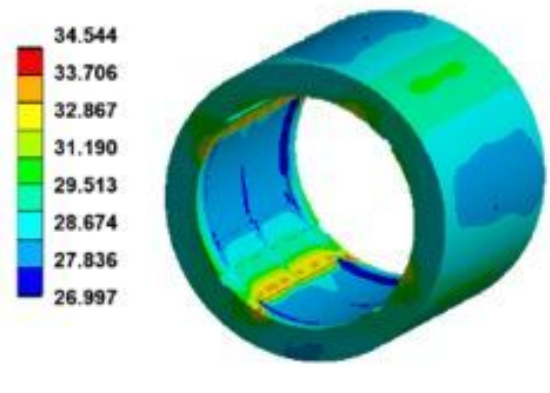

a) $\mathrm{n}=1000 \mathrm{rpm}$

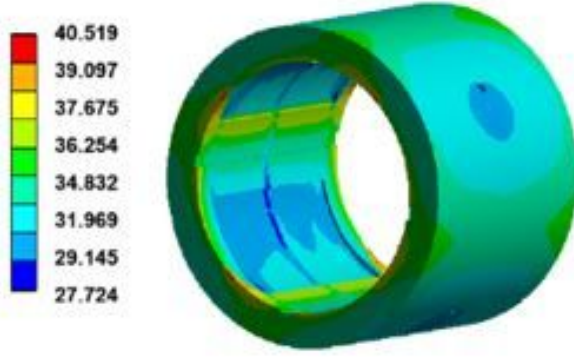

d) n-1800 rpm

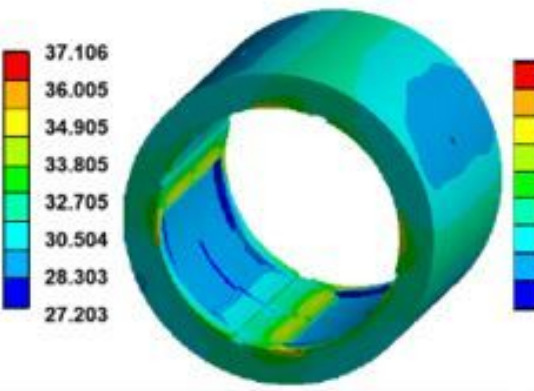

b) $\mathrm{n}=1200 \mathrm{rpm}$

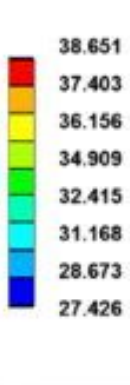

38.651 37.403 36.156 .909 2.415 1.168 8.673 7.426

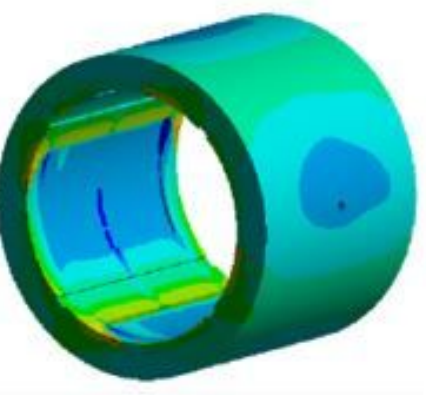

c) $\mathrm{n}=1500 \mathrm{rpm}$

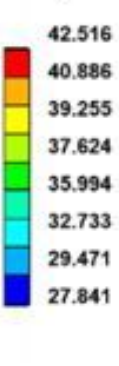

40.886

39.255

3.624

.471

841

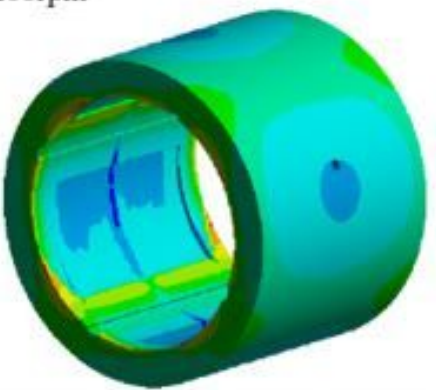

e) n-2000rpm

b

\section{Figure 8}

Temperature cloud diagram of radial bearing pad: (a) Radial bearing bush temperature cloud diagram at constant viscosity, (b) Radial bearing bush temperature cloud diagram with viscosity change 


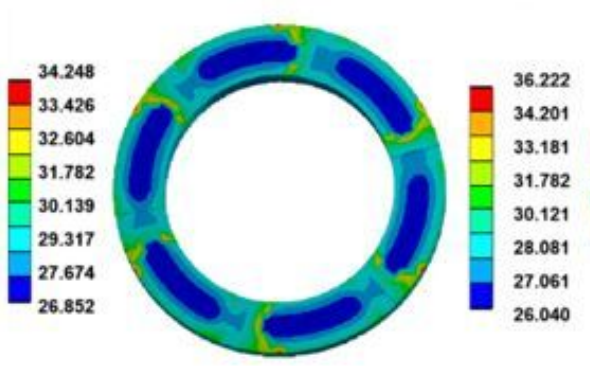

a) $\mathrm{n}=1000 \mathrm{rpm}$

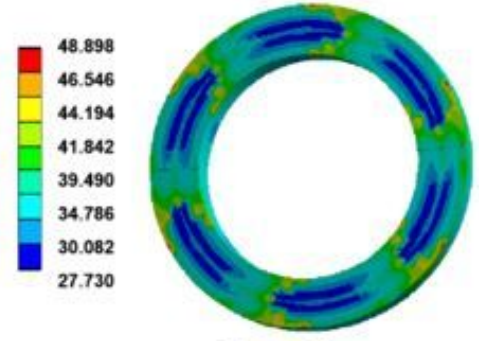

d) $\mathrm{n}=1800 \mathrm{rpm}$

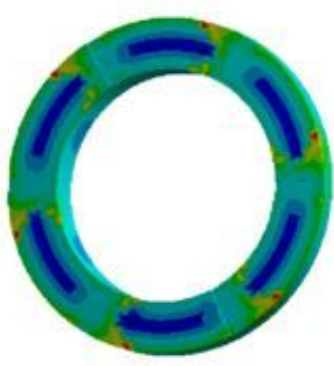

b) $\mathrm{n}=1200 \mathrm{rpm}$

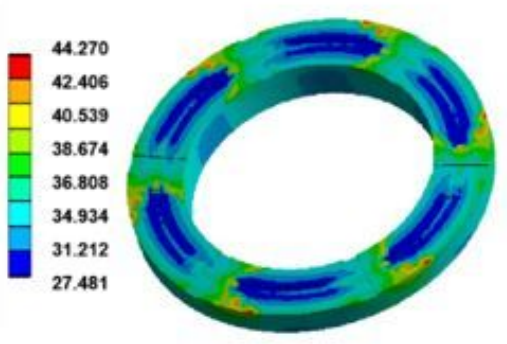

c) $\mathrm{n}=1500 \mathrm{rpm}$

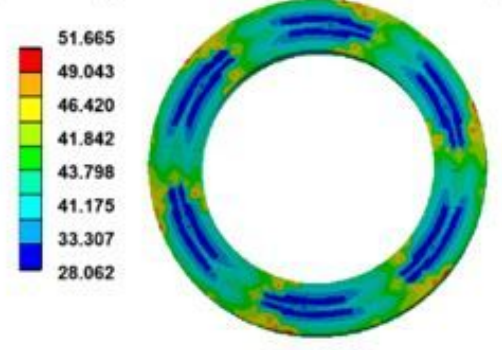

e) $\mathrm{n}=2000 \mathrm{rpm}$

a

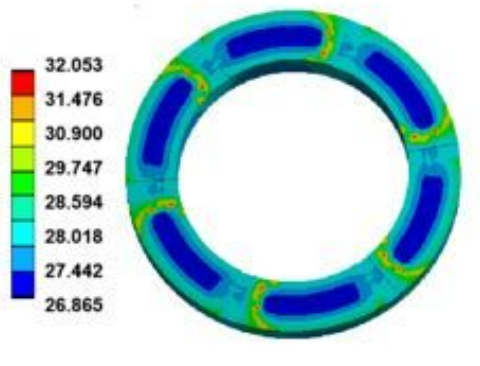

a) $n=1000 \mathrm{rpm}$

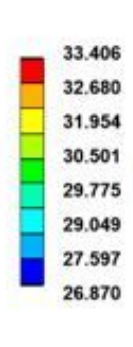

b) $n=1200 \mathrm{rpm}$

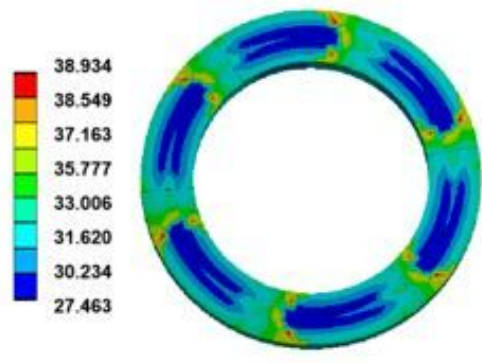

c) $\mathrm{n}=1500 \mathrm{rpm}$

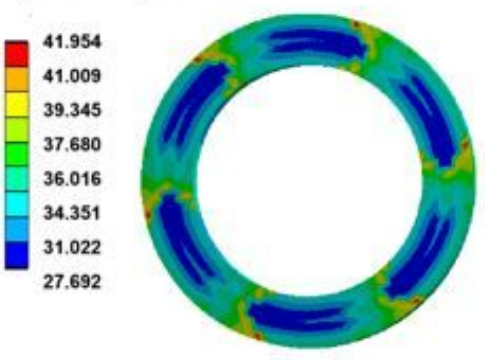

d) $n=1800 \mathrm{rpm}$

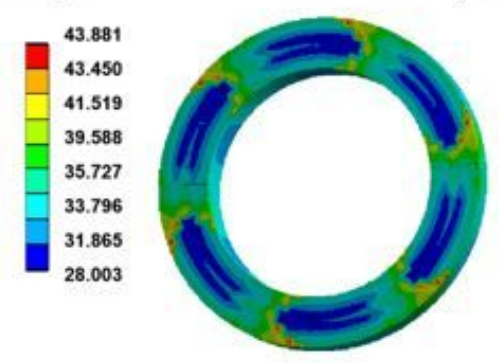

e) $n=2000$ rpm

b

Figure 9

Temperature map of thrust bearing bush: (a) Thrust bearing temperature cloud diagram at constant viscosity, (b) Thrust bearing temperature cloud diagram when viscosity changes 


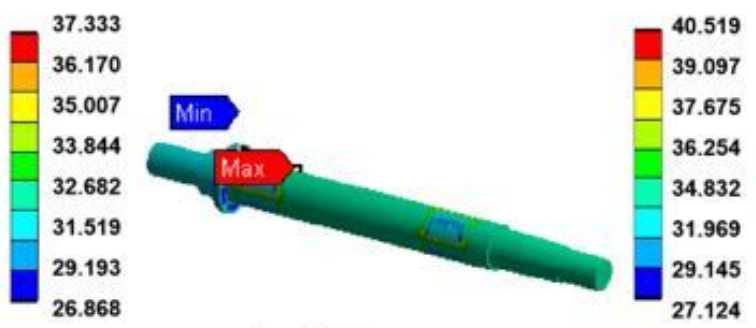

a) $\mathrm{n}=1000 \mathrm{rpm}$

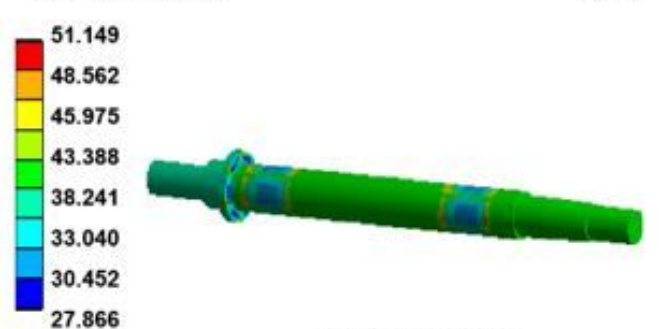

d) $\mathrm{n}=1800 \mathrm{rpm}$

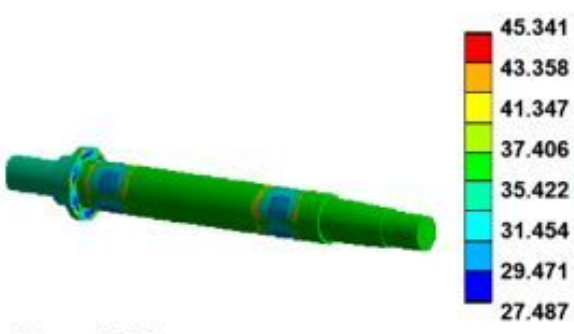

b) $\mathrm{n}=1200 \mathrm{rpm}$

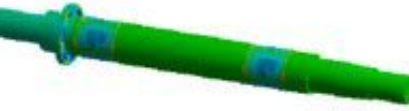

c) $\mathrm{n}=1500 \mathrm{rpm}$

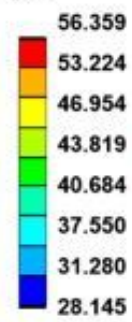

28.145

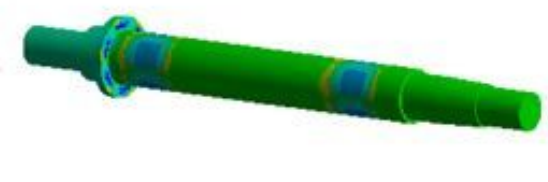

e) $n=2000 \mathrm{rpm}$

a

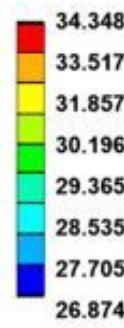

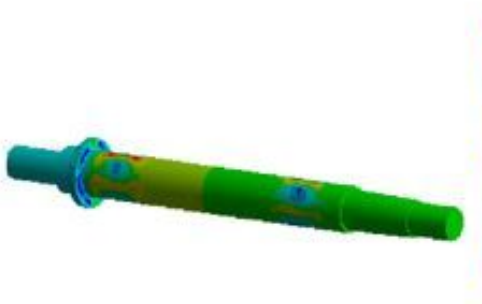

a) $\mathrm{n}=1000 \mathrm{rpm}$

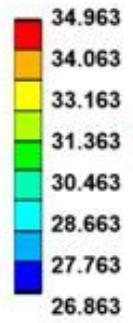

26.863 b) $\mathrm{n}=1200 \mathrm{rpm}$

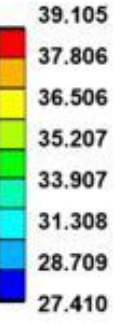

27.410

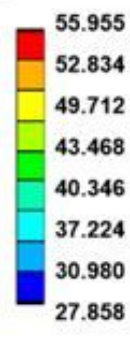

d) $\mathrm{n}=1800 \mathrm{rpm}$

b

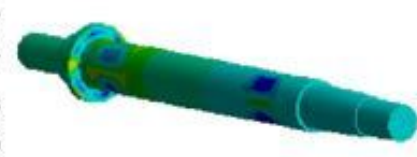

c) $\mathrm{n}=1500 \mathrm{rpm}$

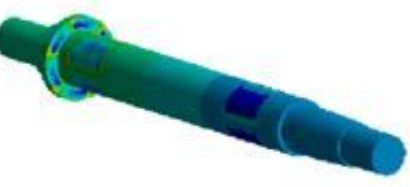

Figure 10

Temperature cloud diagram of the hydrostatic spindle: (a) Shaft temperature cloud diagram at constant viscosity, (b) Shaft temperature cloud diagram at viscosity change 

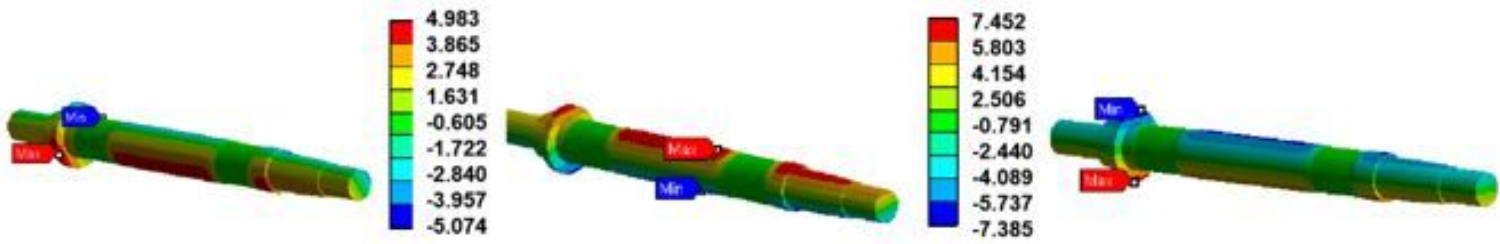

a) $\mathrm{n}=1000 \mathrm{rpm}$

b) $\mathrm{n}=1200 \mathrm{rpm}$

c) $\mathrm{n}=1500 \mathrm{rpm}$
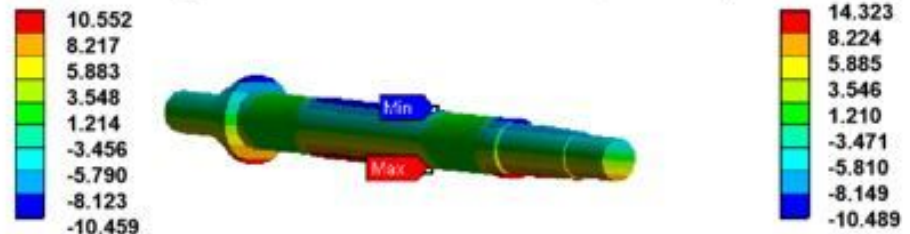

d) $\mathrm{n}=1800 \mathrm{rpm}$

e) $n=2000 \mathrm{rpm}$

a
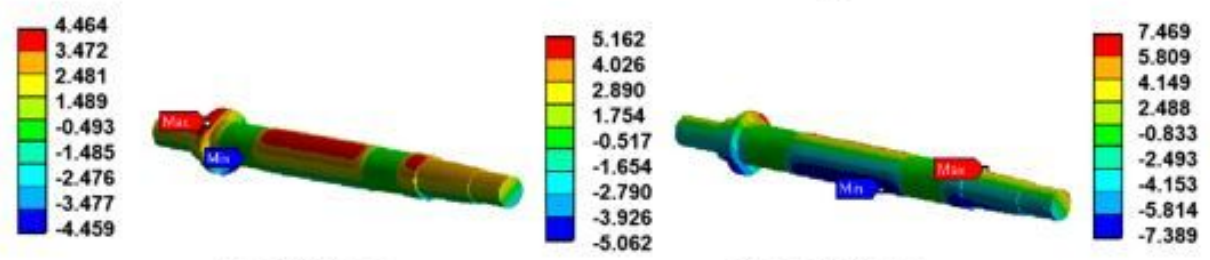

a) $\mathrm{n}=1000 \mathrm{rpm}$

b) $\mathrm{n}=1200 \mathrm{rpm}$

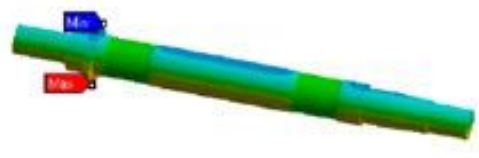

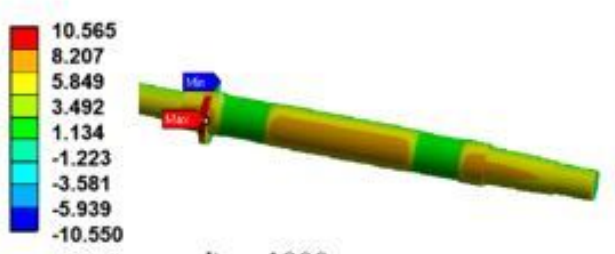

d) $n=1800 \mathrm{rpm}$

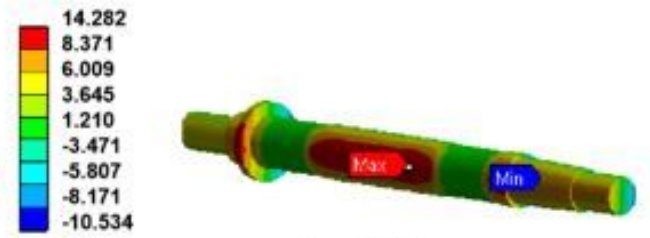

e) $n=2000 \mathrm{rpm}$

b

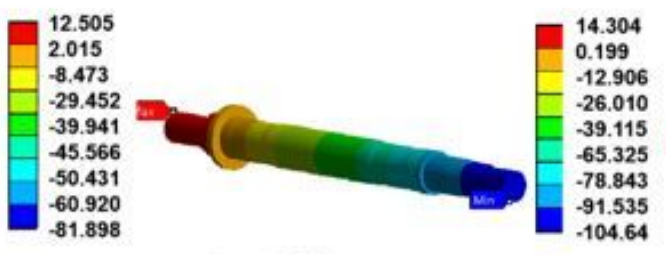

a) $\mathrm{n}=1000 \mathrm{rpm}$

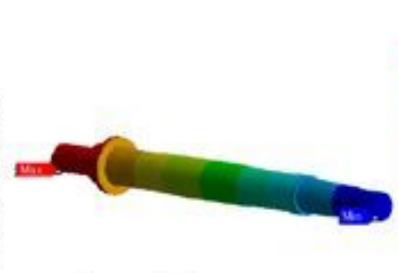

b) $n=1200 \mathrm{rpm}$

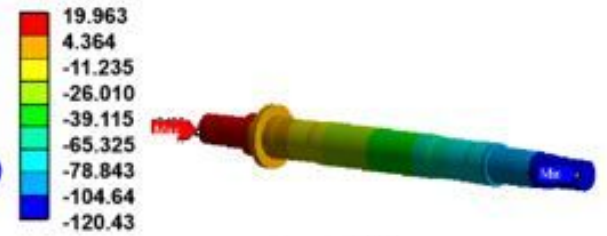

c) $\mathrm{n}=1500 \mathrm{rpm}$

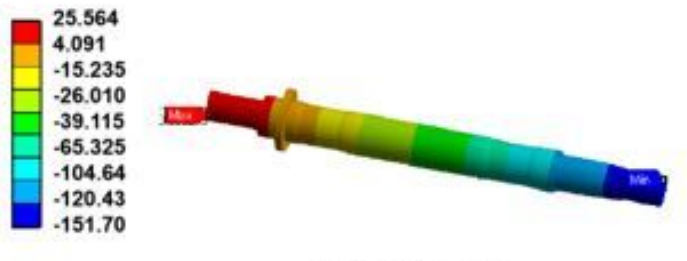

d) $\mathrm{n}=1800 \mathrm{rpm}$

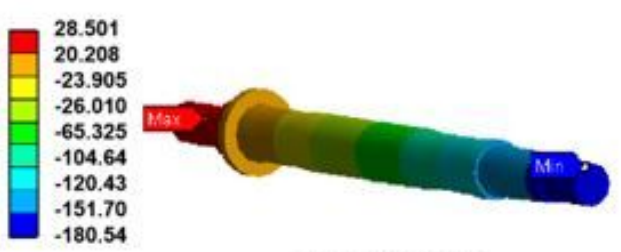

e) $n=2000 \mathrm{rpm}$

\section{Figure 11}

Thermal deformation of spindle shaft when viscosity is fixed: (a) Shaft-X thermal deformation, (b) Shaft$Y$ thermal deformation, (c) Shaft-Z thermal deformation 

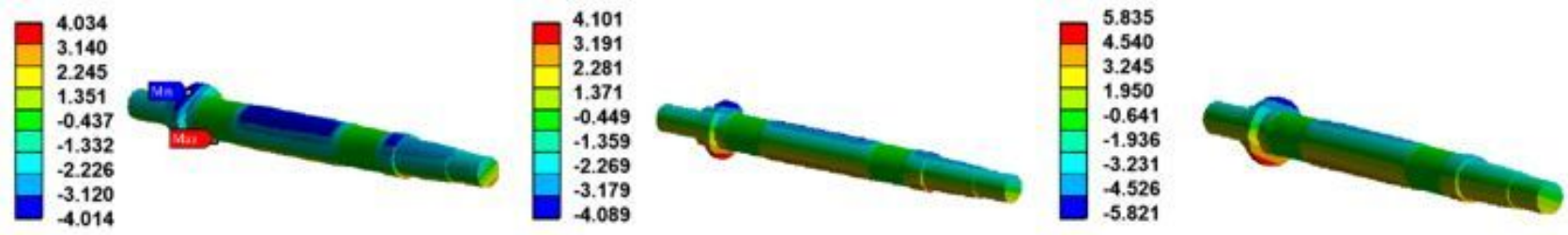

a) $n=1000 \mathrm{rpm}$

b) $\mathrm{n}=1200 \mathrm{rpm}$

c) $\mathrm{n}=1500 \mathrm{rpm}$

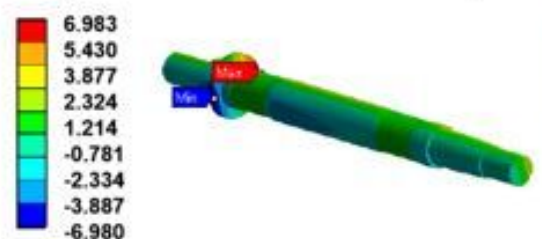

d) $\mathrm{n}=1800 \mathrm{rpm}$

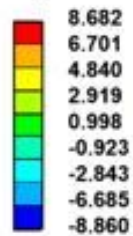

$-8.860$

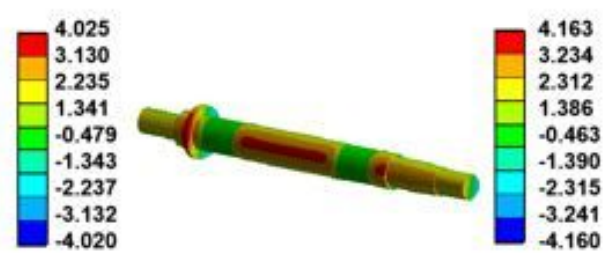

a) $\mathrm{n}=1000 \mathrm{rpm}$

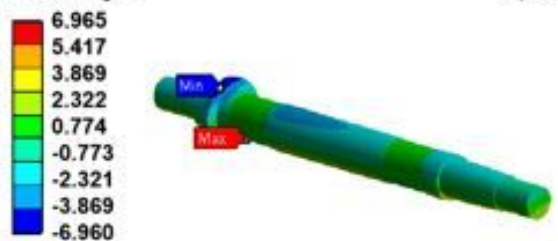

d) $\mathrm{n}=1800 \mathrm{rpm}$

a

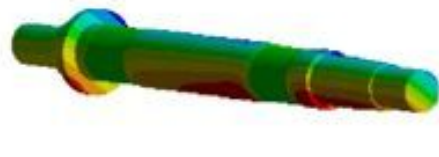

e) $\mathrm{n}=2000 \mathrm{rpm}$

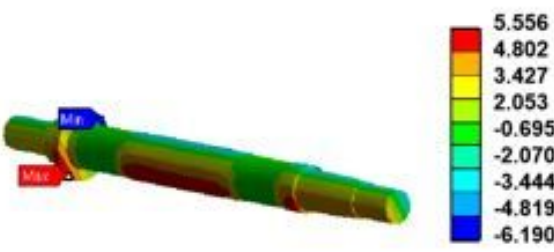

b) $\mathrm{n}=1200 \mathrm{rpm}$
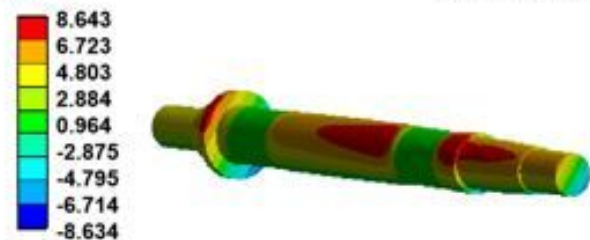

e) $\mathrm{n}=2000 \mathrm{rpm}$

b
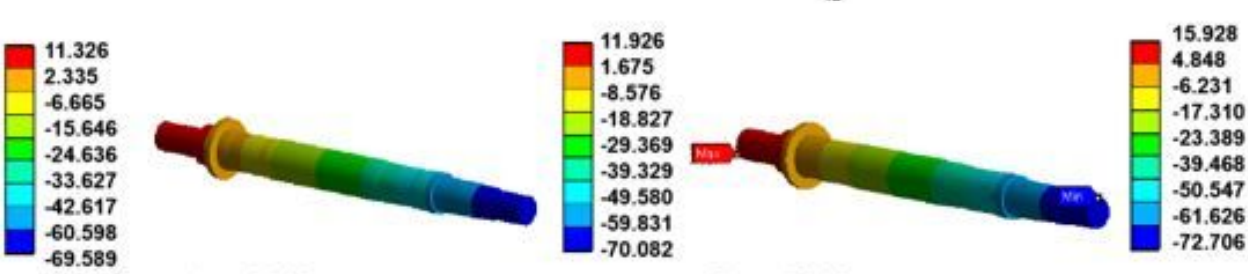

c) $\mathrm{n}=1500 \mathrm{rpm}$

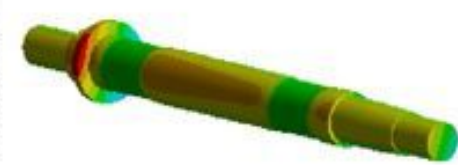

a) $\mathrm{n}=1000 \mathrm{rpm}$

b) $n=1200 \mathrm{rpm}$

c) $\mathrm{n}=1500 \mathrm{rpm}$

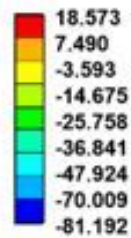

d) $\mathrm{n}=1800 \mathrm{rpm}$

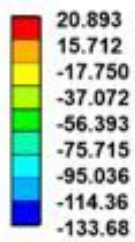

-114.36
-133.68

c

Figure 12

Thermal deformation of spindle shaft under viscosity-temperature variation: (a) Shaft temperature cloud diagram at constant viscosity, (b) Shaft temperature cloud diagram at viscosity change 


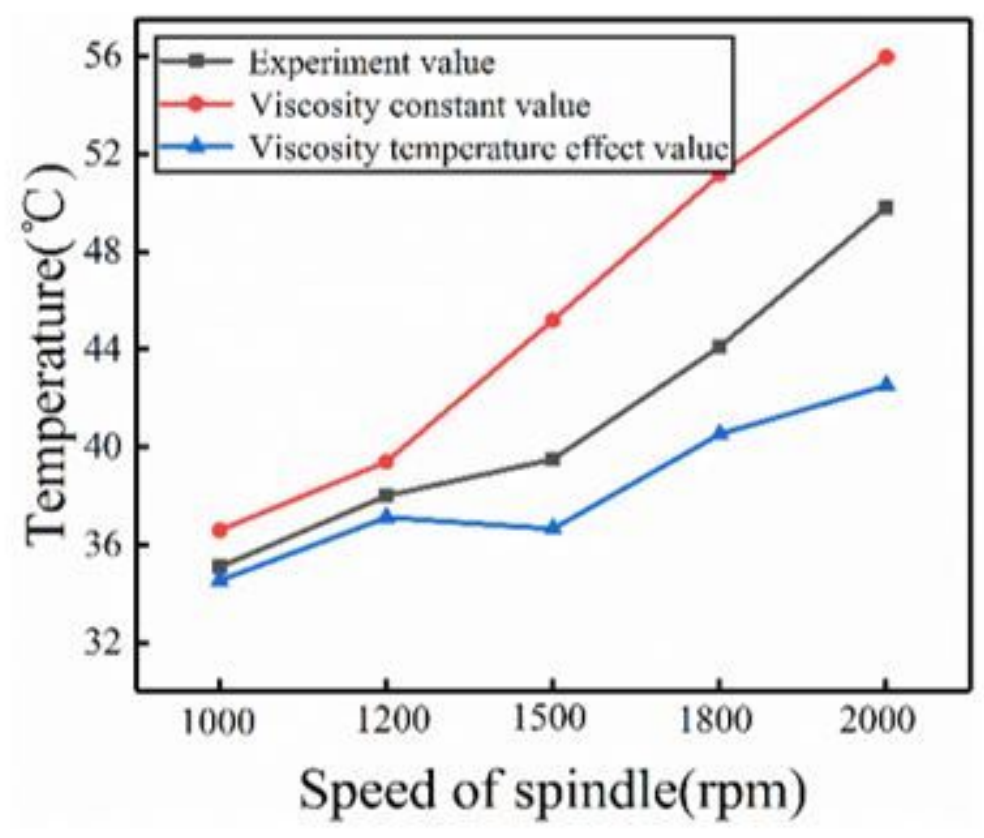

a

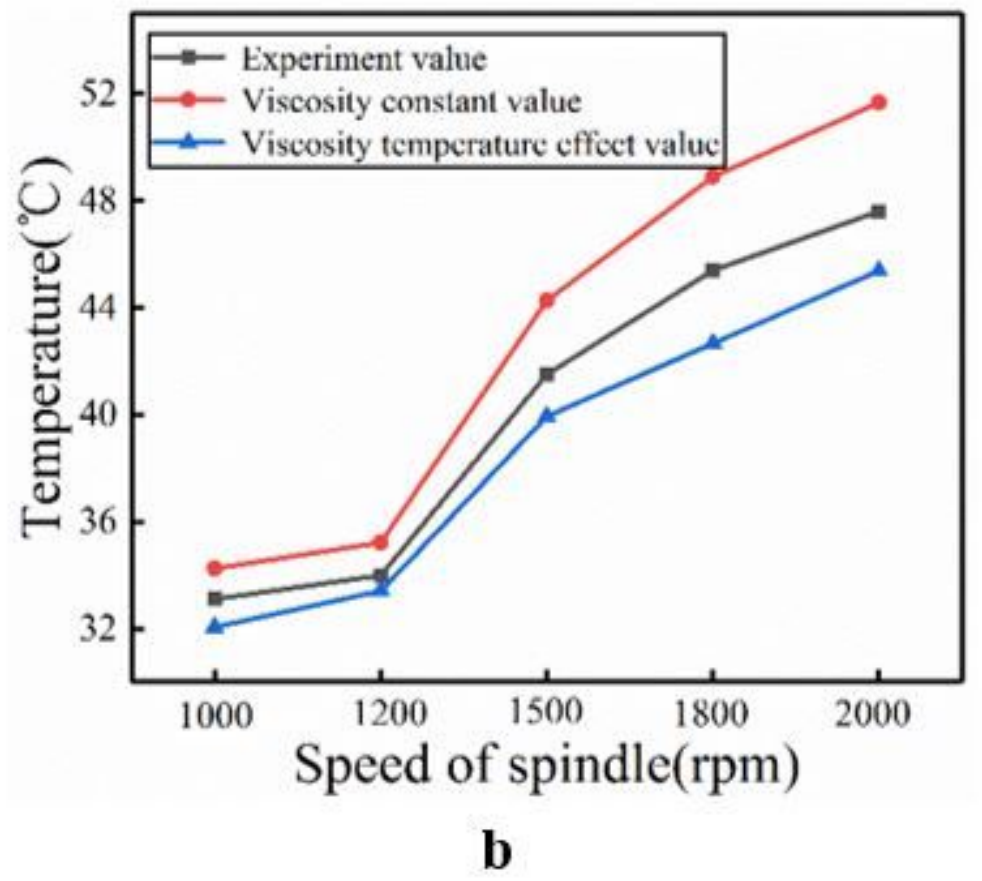

Figure 13

Bearing temperature of hydrostatic bearing: (a) Radial bearing temperature, (b) Thrust bearing temperature 


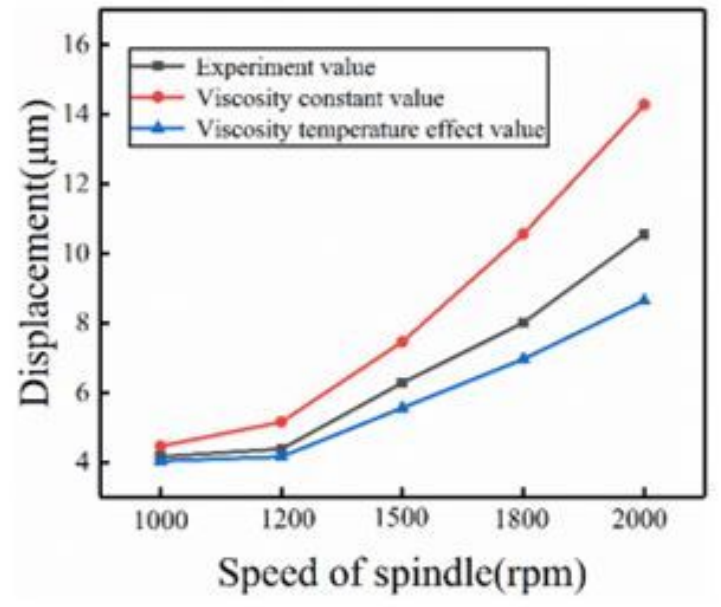

a

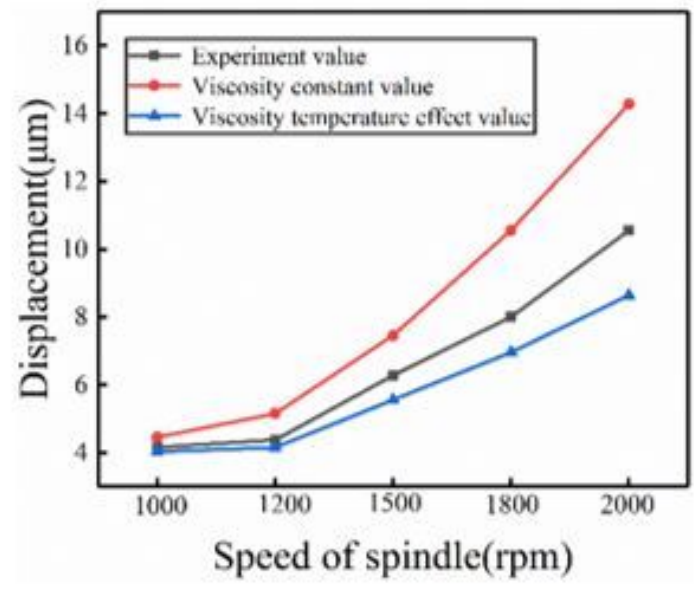

b

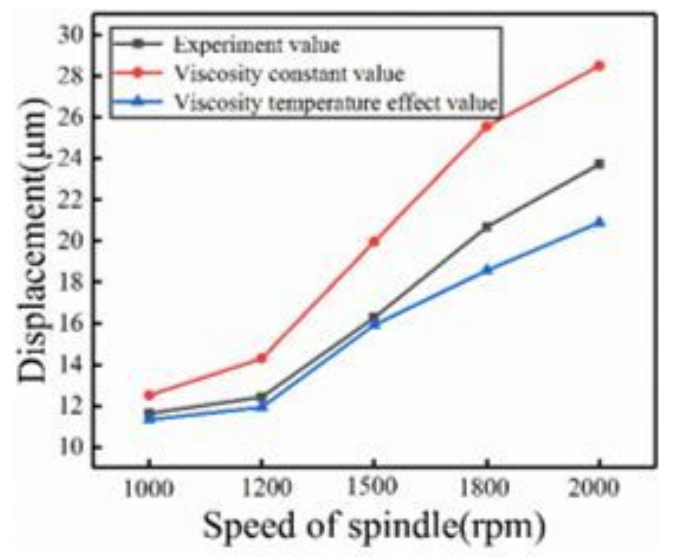

c

Figure 14

Deformation of the hydrostatic spindle shaft: (a) Thermal deformation in the shaft $X$ direction, (b) Thermal deformation in the shaft $Y$ direction, (c) Thermal deformation in the $Z$ direction of the shaft 

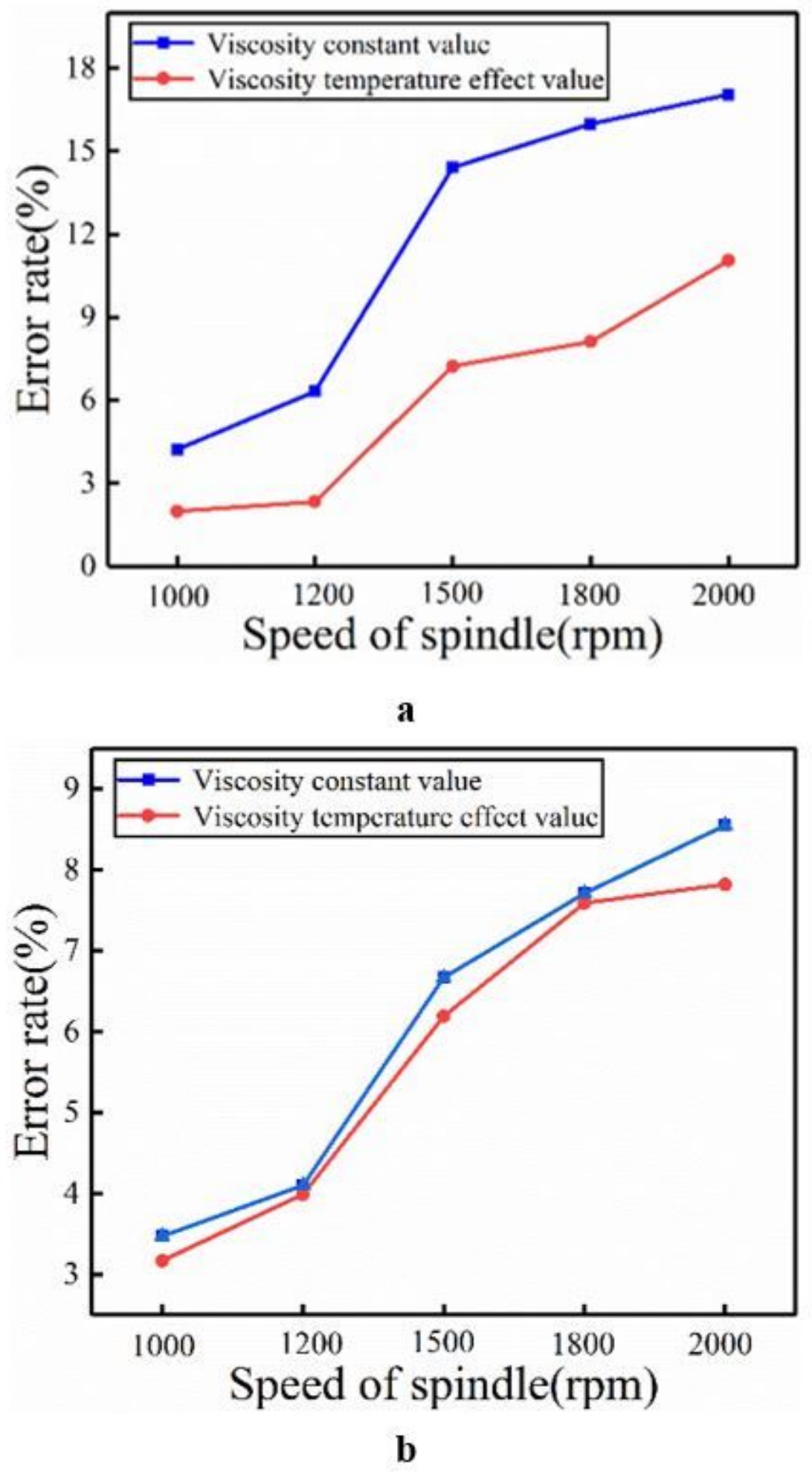

Figure 15

Temperature error analysis of hydrostatic bearing bush: (a) Radial bearing temperature error, (b) Thrust bearing temperature error 


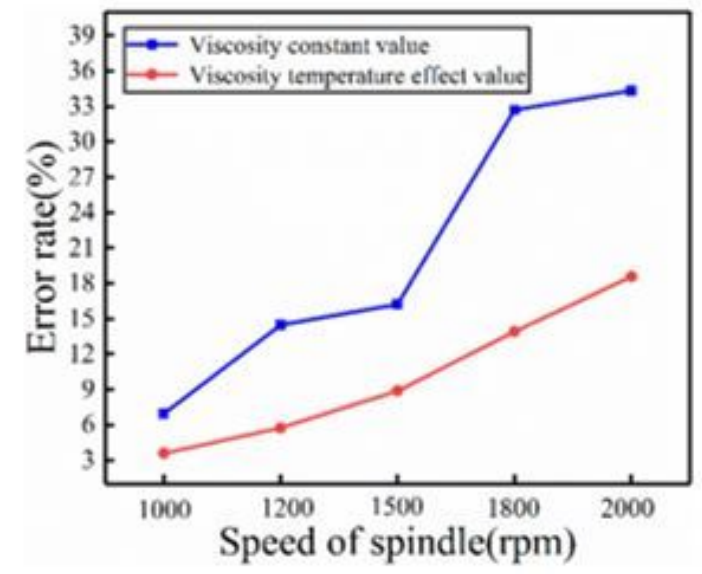

a

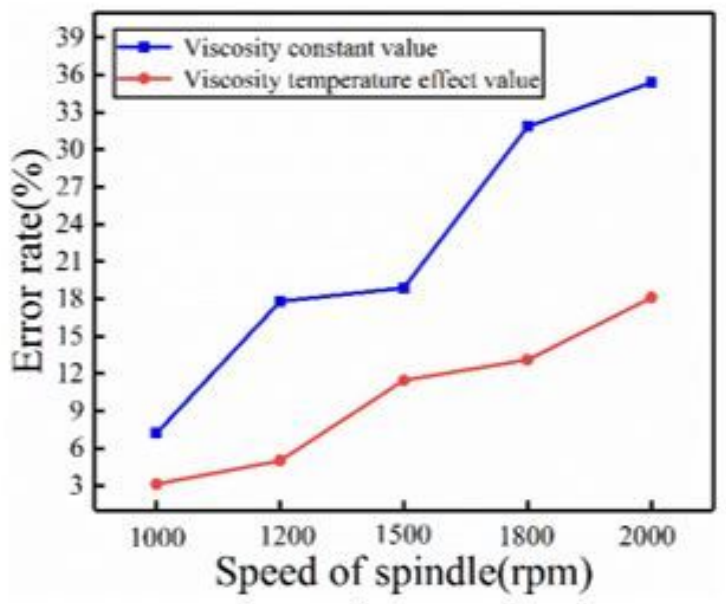

b

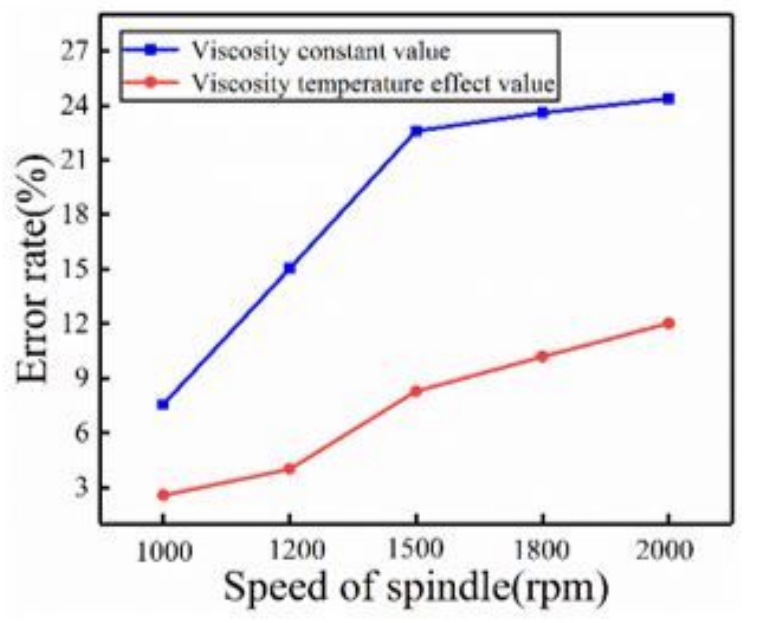

c

\section{Figure 16}

Error analysis of temperature difference of main shaft shaft deformation, (a)Shaft X-direction thermal error, (b) Shaft Y-direction thermal error, (c) Thermal error in the $Z$ direction of the shaft 